\title{
PAK1 Positively Regulates Oligodendrocyte Morphology and Myelination
}

\author{
Tanya L. Brown, ${ }^{1,2}$ Hirokazu Hashimoto, ${ }^{1}{ }^{\oplus}$ Lisbet T. Finseth, ${ }^{1}$ Teresa L. Wood, ${ }^{3}$ and ${ }^{\circledR}$ Wendy B. Macklin ${ }^{1}$ \\ ${ }^{1}$ Department of Cell and Developmental Biology and, ${ }^{2}$ Cell Biology, Stem Cells, and Development Graduate Program, University of Colorado School \\ of Medicine, Aurora, Colorado 80045, and ${ }^{3}$ Department of Pharmacology, Physiology, and Neuroscience, New Jersey Medical School, Rutgers \\ University, Newark, New Jersey 07101
}

The actin cytoskeleton is crucial for oligodendrocyte differentiation and myelination. Here we show that p21-activated kinase 1 (PAK1), a well-known actin regulator, promotes oligodendrocyte morphologic change and myelin production in the CNS. A combination of in vitro and in vivo models demonstrated that PAK1 is expressed throughout the oligodendrocyte lineage with highest expression in differentiated oligodendrocytes. Inhibiting PAK1 early in oligodendrocyte development decreased oligodendrocyte morphologic complexity and altered F-actin spreading at the tips of oligodendrocyte progenitor cell processes. Constitutively activating AKT in oligodendrocytes in male and female mice, which leads to excessive myelin wrapping, increased PAK1 expression, suggesting an impact of PAK1 during active myelin wrapping. Furthermore, constitutively activating PAK1 in oligodendrocytes in zebrafish led to an increase in myelin internode length while inhibiting PAK1 during active myelination decreased internode length. As myelin parameters influence conduction velocity, these data suggest that PAK1 may influence communication within the CNS. These data support a model in which PAK1 is a positive regulator of CNS myelination.

Key words: actin; cytoskeleton; myelin; oligodendrocyte; PAK1

\section{Significance Statement}

Myelin is a critical component of the CNS that provides metabolic support to neurons and also facilitates communication between cells in the CNS. Recent data demonstrate that actin dynamics drives myelin wrapping, but how actin is regulated during myelin wrapping is unknown. The authors investigate the role of the cytoskeletal modulator PAK1 during differentiation and myelination by oligodendrocytes, the myelinating cells of the CNS. They demonstrate that PAK1 promotes oligodendrocyte differentiation and myelination by modulating the cytoskeleton and thereby internode length, thus playing a critical role in the function of the CNS.

\section{Introduction}

Polarized membrane production is a complex biological task. In the CNS, oligodendrocytes produce massive amounts of polarized plasma membrane as they generate myelin that wraps around axons. Myelin provides an insulating sheath that facilitates axon conduction velocity and provides metabolic support to axons (Siegel and Agranoff, 1999; Lee et al., 2012). Oligodendrocytes must integrate signals from the extracellular

Received Jan. 29, 2020; revised Jan. 4, 2021; accepted Jan. 9, 2021.

Author contributions: T.L.B., T.L.W., and W.B.M. designed research; T.L.B., H.H., and L.T.F. performed research; T.L.B. and W.B.M. analyzed data; T.L.B. wrote the first draft of the paper; H.H., L.T.F., T.L.W., and W.B.M. edited the paper.

This work was supported by National Science Foundation GRFP DGE-1553798 to T.L.B. and National Institutes of Health R37 \#82203 to W.B.M. and T.L.W. We thank members of the W.B.M. and T.L.W. laboratories for valuable discussion and feedback on this manuscript.

The authors declare no competing financial interests.

Correspondence should be addressed to Wendy B. Macklin at wendy.macklin@ucdenver.edu.

https://doi.org/10.1523/JNEUROSCI.0229-20.2021

Copyright $\odot 2021$ the authors environment, nearby cells, and the axons themselves to determine how much myelin to produce (Kirby et al., 2006; Hughes et al., 2013; Hines et al., 2015; Mensch et al., 2015). Recent data highlight the complex role of the actin cytoskeleton throughout oligodendrocyte development. Actin polymerization is required for oligodendrocyte precursor cell (OPC) migration as OPCs extend and retract filopodial-like processes, integrating signals from the surrounding environment (Liang et al., 2004; Nawaz et al., 2015; Zuchero et al., 2015). OPCs then differentiate into oligodendrocytes where they contact axons and begin producing myelin. Myelin wrapping, the process of extending membrane around an axon, is driven by the cycle of actin polymerization and depolymerization at the inner tongue, also known as the leading edge, of the myelin wrap (Nawaz et al., 2015; Zuchero et al., 2015). F-actin localizes to the leading edge during myelin wrapping, expanding the cytoplasm that protrudes underneath previous myelin wraps and between those wraps and the axon, driving membrane around the axon. F-actin is immediately depolymerized, allowing for myelin compaction behind the 
leading edge (Nawaz et al., 2015). The signals that regulate cytoskeletal dynamics during oligodendrocyte differentiation and myelination are unknown.

The signaling pathways that regulate myelin production (i.e., myelination) by oligodendrocytes have been intensively investigated. Most studies define myelin production by myelin thickness or the number of myelinated axons. The AKT/mTOR and the MEK/ERK pathways, as well as modulators of the actin cytoskeleton, positively regulate myelin thickness. Conditional KOs of the AKT/mTOR or the MEK/ERK pathways decrease myelin thickness, whereas activating these pathways increases myelin thickness (Flores et al., 2008; Narayanan et al., 2009; Goebbels et al., 2010; Ishii et al., 2012, 2013, 2019; Bercury et al., 2014; Wahl et al., 2014). Altering actin cytoskeletal dynamics by increasing actin depolymerization increases myelin thickness (Zuchero et al., 2015), whereas conditionally deleting ADF/cofilin, an actindepolymerizing protein, leads to a decrease in myelin thickness (Nawaz et al., 2015). These studies establish that these pathways regulate myelin thickness and increasing myelin wrapping. Little is known about how they affect myelin internode length, an important and distinct parameter that influences axonal conduction velocity. Additionally, less is known about the regulation of the actin cytoskeleton during myelination, compared with the AKT or ERK signaling pathways.

The p21-activated kinase (PAK) family of serine/threonine kinases regulates multiple signaling pathways, some of which are involved in myelination. PAKs are known to act both upstream and downstream of multiple pathways, including the AKT/ mTOR, MEK/ERK, and cytoskeletal pathways, each of which regulates oligodendrocyte development and myelination (Frost et al., 1997; Slack-Davis et al., 2003; Higuchi et al., 2008). Group I PAKs (PAK1-3) share a high degree of sequence identity and are characterized by a Cdc42/Racl interacting binding domain (Zenke et al., 1999; Tang et al., 2000; Papakonstanti and Stournaras, 2002). PAK3 is expressed predominately in the brain, and recent data indicate that PAK3 regulates oligodendrocyte differentiation and myelination (Manser et al., 1994; Maglorius Renkilaraj et al., 2017). Additionally, global loss of PAK1 and PAK3 decreases total myelin production in the brain, suggesting that PAK1 may be important for myelination (Huang et al., 2011). Given its high expression in oligodendrocyte lineage cells (Y. Zhang et al., 2014; Marques et al., 2016) and its known role regulating signaling pathways involved in oligodendrocyte differentiation, we tested the hypothesis that PAK1 is required for oligodendrocyte differentiation and myelination. Our studies establish that PAK1 increases in expression throughout oligodendrocyte differentiation and during excessive myelin wrapping, and it positively regulates myelin production by modulating F-actin at the edge of OPC processes.

\section{Materials and Methods}

Zebrafish maintenance and live imaging. All zebrafish experiments completed in this study were approved by the Institutional Animal Care and Use Committee at the University of Colorado School of Medicine. Embryos were raised at $28.5^{\circ}$ on a $14 / 10$ light cycle in embryo media $(\mathrm{EM})\left(250 \mathrm{ml} 20 \times \mathrm{EM}\right.$ stock: $\mathrm{NaCl} 17.5 \times \mathrm{g}, \mathrm{KCl} 0.75 \times g, \mathrm{CaCl}_{2} 2 \mathrm{H}_{2} \mathrm{O}$ $2.9 \times g, \mathrm{KH}_{2} \mathrm{HPO}_{4} 0.41 \times g, \mathrm{NaHPO}_{4}-2 \mathrm{H}_{2} \mathrm{O} 0.178 \times g, \mathrm{MgSO}_{4}-7 \mathrm{H}_{2} \mathrm{O}$ $4.9 \times g, \mathrm{dH}_{2} \mathrm{O}$ to $1000 \mathrm{ml}$ ), sodium bicarbonate $0.3 \times g, \mathrm{dH}_{2} \mathrm{O}$ to $5 \mathrm{~L}$ and staged according to hours post-fertilization (hpf), days post-fertilization (dpf), and morphologic criteria (Kimmel et al., 1995). Zebrafish were anesthetized using tricaine (MS-222). For imaging, live embryos were mounted laterally in $1 \%$ low-melt agarose and tricaine and imaged directed above the yolk sac extension on a Leica Microsystems DM-6000 confocal with a $25 \times$ water immersion lens. Individual myelin internodes were traced and analyzed in 3D using IMARIS image analysis software (Bitplane). Image brightness and contrast were increased uniformly in individual images to provide a clear image.

$q P C R$. Zebrafish embryos were collected for RNA analysis in treatment groups of 20 fish. They were anesthetized with ethyl 3-aminobenzoate methanesulfonate salt (tricaine) (Sigma Millipore, \#E10521-10G, catalog \#866-86-2), collected into $1.5 \mathrm{ml}$ tubes, liquid was removed, snap frozen in liquid nitrogen, and stored at $-80^{\circ}$ in Trizol Reagent (Thermo Fisher Scientific \#15596026). RNA was isolated using Direct-zol RNA miniprep Kit plus DNase treatment (Zymo Research, \#R2072). After isolation, $100 \mathrm{ng} / \mu \mathrm{l}$ of mRNA was reverse-transcribed using iScript Reverse Transcription Supermix for qRT-PCR (Bio-Rad, \#1708841). qRT-PCR was performed using TaqMan Universal PCR Master Mix (Thermo Fisher Scientific, \#4304437) on a StepOnePlus realtime PCR (Applied Biosystems, \#4376600). TaqMan probes were ordered through Thermo Fisher Scientific, $m p z$ (Dr03131915_m1), 36k (flj13639) (Dr034338676_m1), and reference genes gapdh (Dr03436842_m1), and rpl13 (Dr03101114_g1). mbp (jh71 mbp) was designed through Eurofins using the following sequences: forward 5' -GTTCTTCGGAGGAGACAAGAAGAG-3', reverse 5' GTCTCTGTGGAGAGGAGGATAGATGA-3'. All experiments followed the MIQE Guidelines for qPCR (Bustin et al., 2009). Data were analyzed using one-sample $t$ test against the hypothetical control value of 1 for statistical significance $(p<0.05)$

Rat OPCs. All animal experimental protocols were approved by the University of Colorado School of Medicine Animal Care and Use Committee and were conducted in accordance with the National Institutes of Health Guide for the care and use of laboratory animals. Mixed glial cultures were generated from P0-P4 Sprague Dawley rat pups. The cerebra of rat pups were dissected and homogenized in Hanks balanced salt solution following removal of meninges to generate a single cell suspension. Cells were plated into poly-D-lysine-coated or poly-Lornithine-coated flasks and grown in DMEM high glucose with $10 \%$ FBS, $2 \mathrm{~mm}$ L-glutamine and $50 \mathrm{U} / \mathrm{ml}$ Pen/Strep for $10-12 \mathrm{~d}$ with three media changes. Microglia were shaken off for $1 \mathrm{~h}$ at $150 \mathrm{rpm}$ at $37^{\circ} \mathrm{C}$. Media was replaced, and OPCs were purified by shaking overnight at $225 \mathrm{rpm}$ at $37^{\circ} \mathrm{C}$. The media was collected and passed through a $70 \mu \mathrm{m}$ filter. The media was added to uncoated $10 \mathrm{~cm}$ dishes and incubated for $1 \mathrm{~h}$ at $37^{\circ} \mathrm{C}$ to remove astrocytes. Media was transferred to $50 \mathrm{ml}$ Falcon tubes and spun for $4 \mathrm{~min}$ at $1200 \mathrm{rpm}$ to pellet OPCs. OPCs were then resuspended in OPC media (DMEM, $1 \times \mathrm{N} 2,40 \mathrm{~nm}$ D-biotin, $10 \mathrm{~nm}$ hydrocortisone, $0.1 \% \mathrm{BSA}, 50 \mathrm{U} / \mathrm{ml} \mathrm{Pen} /$ Strep, $2 \mathrm{~mm}$ L-glutamine, $10 \mathrm{ng} / \mathrm{ml}$ PDGF, $10 \mathrm{ng} / \mathrm{ml} \mathrm{FGF)} \mathrm{and} \mathrm{plated} \mathrm{onto} \mathrm{acid} \mathrm{washed} \mathrm{glass} \mathrm{cover-}$ slips or coated (poly-D-lysine, fibronectin, laminin) plastic dishes according to experimental protocol.

Mouse OPCs. For mouse OPCs, cortices of P0-P2 mouse pups were dissected; $1 \mathrm{ml}$ of papain solutions and DNase was added and gently dissociated by titration. The mixture was incubated at $37^{\circ} \mathrm{C}$ for $10 \mathrm{~min}$, triturated again; $5 \mathrm{ml}$ OPC media (DMEM, 100× OL supplement; DMEM, BSA $10.2 \mathrm{mg} / \mathrm{ml}$, progesterone $6 \mu \mathrm{g} / \mathrm{ml}$, putrescine $1.61 \mathrm{mg} / \mathrm{ml}$, sodium selenite $500 \mathrm{ng} / \mathrm{ml}$, T3 $40 \mu \mathrm{g} / \mathrm{ml}$; insulin $2.5 \mu \mathrm{g} / \mathrm{ml}$, GlutaMAX, Holotransferrin $50 \mu \mathrm{g} / \mathrm{ml}$, B27 $1 \times$, PDGF $10 \mathrm{ng} / \mathrm{ml}$, FGF $10 \mathrm{ng} / \mathrm{ml}$ ) and $150 \mu \mathrm{l}$ of DNase/brain was added to mixtures, which were incubated for $5 \mathrm{~min}$ at room temperature. Tubes were spun at $300 \times g$ for $5 \mathrm{~min}$. Media was aspirated, and pellets were resuspended in $1 \mathrm{ml}$ of OPC media. Mixture was added to T75 flask with $10 \mathrm{ml} \mathrm{OPC}$ media and placed in $8.5 \% \mathrm{CO}_{2}$ incubator for $24 \mathrm{~h}$. Media was aspirated off and replaced with $10 \mathrm{ml}$ of fresh OPC media. Media was changed on every $2 \mathrm{~d}$. On day 8 , insulin was added to the media. Microglia were shaken off at $100 \mathrm{rpm}$ for $1 \mathrm{~h}$ at room temperature. Media was replaced, and OPCs were purified by shaking overnight at $190 \mathrm{rpm}$ at $37^{\circ} \mathrm{C}$. Media containing cells was collected and filtered through $40 \mu \mathrm{m}$ filter into noncoated Petri plates for microglia attachment for $30 \mathrm{~min}$ to $1 \mathrm{~h}$. Media was placed in $15 \mathrm{ml}$ Falcon tubes and spun at $1200 \mathrm{rpm}$ for $5 \mathrm{~min}$. Supernatant was removed, and cells were resuspended in oligodendrocyte media containing PDGF/FGF. Media was changed every $48 \mathrm{~h}$ (O'Meara et al., 2011). For inhibitor experiments, OPCs were treated with either DMSO or FRAX486 after $2 \mathrm{~d}$ in oligodendrocyte media. 
shRNA. For shRNA experiments in rat OPCs (rOPCs), cells were plated on glass coverslips. Plasmid DNA containing rat PAK1 shRNA (Target 1: TAAAATTCCAACACATCCA; Target 2: TTCCGGGTC AAAGCATCTG; Target 3: ATCCCCGTAAACTCCCCTG; scrambled control: GCTCCACGCTTCACTACCA) was cloned using pSUPER RNAi System (catalog \#VEC-PRT-0002). Plasmid DNA was added to Xfect reaction buffer (Xfect Transfection Reagent, Takara, catalog \#631317). Xfect Polymer was then added to mixture. Mixture was vortexed for $10 \mathrm{~s}$ and incubated at room temperature for $10 \mathrm{~min}$. The mixture was spun in a tabletop centrifuge and then added dropwise to the cell culture medium. The plates were gently tapped and then incubated for $4 \mathrm{~h}$ at $37^{\circ} \mathrm{C}$. Media containing the shRNA was removed by aspiration and replaced with differentiation media, replaced every $2 \mathrm{~d}$. The cells were incubated at $37^{\circ} \mathrm{C}$ for $5 \mathrm{~d}$ until analysis. For shRNA in C6 glioma cells, cells were plated on $6 \mathrm{~cm}$ plastic dishes. Cells were transfected overnight, treated with puromycin for 1 week to select for transfected cells, then collected and plated on $6 \mathrm{~cm}$ dishes (1 million cells/dish), and collected the next day for Western blot for PAK1 (Cell Signaling Technology, 2602) or PAK1 target LIM domain kinase 1 (pLIMK; Cell Signaling Technology, 3841) protein expression.

Immunocytochemistry. rOPCs were collected for analysis after $1 \mathrm{~h}$ incubation with live stain (1:4 O1/O4 antibody [Dai et al., 2015] in OPC media). rOPCs were washed twice with ice-cold PBS and fixed overnight in $500 \mu \mathrm{l}$ of $4 \%$ PFA. Cells were rinsed twice with PBS and stored at $4^{\circ} \mathrm{C}$ for immunocytochemistry. Images were taken using Leica Microsystems DM-6000 confocal with a $25 \times$ water immersion lens or $63 \times$ oil immersion lens. For high magnification, images were taken using Nikon NSIM with $100 \times$ TIRF objective.

Immunohistochemistry. For PAK1 staining, freshly dissected tissue was obtained from P8 mice, embedded in Tissue-Tek Optimal Cutting Temperature compound (Sakura Finetech, \#4583) and quickly frozen in liquid nitrogen. Brains were sectioned at $5 \mu \mathrm{m}$ thickness by a cryostat (Leica Biosystems, CM1950) and thaw-mounted on slides. These sections were fixed with cold acetone $\left(-30^{\circ} \mathrm{C}\right)$ for $20 \mathrm{~min}$, then blocked for $1 \mathrm{~h}$ in blocking solution (5\% normal donkey serum, $0.1 \%$ Triton X-100 in PBS). Sections were incubated in primary antibodies diluted in blocking solution $24 \mathrm{~h}$ at $4^{\circ} \mathrm{C}$. Primary antibodies used are as follows: rabbit anti-PAK1 (1:200, Abcam, ab131522, RRID:AB_11156726) and goat anti-SOX10 (1:500, R\&D Systems, AF2864, RRID:AB_442208). After washing with PBS, the sections were incubated in biotin-conjugated anti rabbit IgG diluted in blocking buffer overnight at $4^{\circ} \mathrm{C}$. After washing with PBS-T (0.1\% Triton X-100/PBS), sections were incubated for $2 \mathrm{~h}$ at room temperature with AlexaFluor-488-conjugated Donkey anti-Rabbit and AlexaFluor-594-conjugated Donkey anti-Goat (Jackson ImmunoResearch Laboratories) diluted in blocking buffer. After washing with PBS-T $(0.1 \%$ Triton X-100/PBS), the sections were coverslipped in Fluoromount G (Southern Biotechnology). Images were taken with a Leica Microsystems DM-6000 confocal with a $63 \times$ oil immersion lens.

For AKT-DD animal staining, mice were anesthetized with Fatal Plus, and tissue was fixed by transcardial perfusion with PBS followed by $4 \%$ PFA at $4^{\circ} \mathrm{C}$. Brain was dissected, postfixed overnight, and transferred to cryoprotection solution ( $30 \%$ sucrose in PBS) overnight at $4^{\circ} \mathrm{C}$. Freefloating $30 \mu \mathrm{m}$ coronal sections were prepared on a cryostat (Leica Microsystems, CM1950), and sections were stored at $4^{\circ} \mathrm{C}$ in cryostorage solution (30\% ethylene glycol, $30 \%$ sucrose, and 1\% PVP-40 in $0.1 \mathrm{M}$ Sorenson's buffer). Free-floating sections were washed in PBS, and antigen retrieval was performed with $10 \mathrm{~mm}$ sodium citrate, $\mathrm{pH} 6.0$, plus $0.05 \%$ Tween 20 for $10 \mathrm{~min}$ at $550 \mathrm{~W}$ in a PELCO BioWave Pro tissue processor (Ted Pella), followed by washing in PBS. They were blocked for $1 \mathrm{~h}$ in blocking solution ( $5 \%$ normal donkey serum, $0.1 \%$ Triton X100 in PBS). Sections were incubated in primary antibodies diluted in blocking solution $48 \mathrm{~h}$ at $4^{\circ} \mathrm{C}$. Primary antibodies used are as follows: rabbit anti-PAK1 (1:500, Cell Signaling Technology, \#2602, RRID:AB_ 330222), goat anti-GFP (1:500, Abcam, ab6673, RRID:AB_305643), and rat anti-PLP1 (clone AA3) (Yamamura et al., 1991). After washing with PBS, the sections were incubated in biotin-conjugated anti-rabbit IgG diluted in blocking buffer overnight at $4^{\circ} \mathrm{C}$. After washing with PBS, sections were incubated for $2 \mathrm{~h}$ at room temperature with AlexaFluor-488conjugated Donkey anti-Goat and Rat IgG (Jackson ImmunoResearch
Laboratories), and AlexaFluor-594-conjugated streptavidin (Jackson ImmunoResearch Laboratories) diluted in blocking buffer. After washing with PBS, the sections were mounted on slides and coverslipped in Fluoromount G (Southern Biotechnology). Images were taken Leica Microsystems DM-6000 confocal with a $25 \times$ water immersion lens.

Cloning. The tol2kit was used to construct plasmids (Kwan et al., 2007). pEXPR-myrf:PAK1 and pEXPR:mbp:PAK1-EGFP constructs were created by subcloning the appropriate PAK1 constructs into the pME plasmid, which was then used for recombination with p5E-myrf or p5E-mbp, p3E-2AnlsmCherry, and pDEST-nonet plasmids using the Tol 2 kit. The resulting plasmids were verified by restriction digest and sequencing.

Plasmid list. The plasmid list is as follows: P5E-mbp; P5E-myrf (a gift from Bruce Appel); pME-p3E-2AnlsmCherry; pEBG-PAK1 83-149 (a gift from Jonathan Chernoff, Addgene plasmid \#12214); pEBG-PAK1 83-149 L107F (a gift from Jonathan Chernoff, Addgene plasmid \#12215); pCMV6M-PAK1 K299R (a gift from Jonathan Chernoff, Addgene plasmid \#12210); pCMV6M-PAK1 T423E (a gift from Jonathan Chernoff, Addgene plasmid \#12208); pDEST-nonet; pDEST-no heart marker; pEXPR-mbp: mEGFP; pEXPR-myrf:PAK1 autoinhibitory domain (AID); pEXPR-myrf: PAK1 AID L107F; pEXPR-myrf:PAK1 T423E; pEXPR-myrf:PAK1 K299R; and pEXPR:mbp:PAK1-EGFP.

Drug inhibitor experiments. Pharmaceuticals used to study PAK1 impact on myelin gene expression were FRAX486 (Tocris Bioscience, \#5190, catalog \#1232030-35-1), NVS PAK1 1 (Tocris Bioscience, \#6132, catalog \#1783816-74-9), and G5555 (Tocris Bioscience, \#6051, catalog \#1648863-90-4). All pharmaceuticals were reconstituted in DMSO. For zebrafish experiments, each pharmaceutical concentration was applied in $1 \%$ DMSO in EM, with a sample group of 20 embryos. All conditions were performed in 3-5 biological replicates. Pharmaceuticals diluted in EM were applied at $2 \mathrm{dpf}$, refreshed at $3 \mathrm{dpf}$, and samples were collected at $4 \mathrm{dpf}$. Drugs were diluted into EM to make working concentrations with a final concentration of $1 \%$ DMSO. Control solutions contained $1 \%$ DMSO in EM. Zebrafish embryos were collected following timed matings. Transgenic embryos were sorted for fluorescence (for internode analysis) or WT embryos (for qPCR analysis), and they were dechorionated and treated with appropriate drug or DMSO control. Drug treatments were initiated at $48 \mathrm{hpf}$ for all experiments. For internode analysis, fish were treated from 48-72 hpf, after which zebrafish were anesthetized using tricaine (MS-222) and live imaged. For qPCR, embryos were treated from 48 to $96 \mathrm{hpf}$ with drug renewal/EM change after $24 \mathrm{~h}$.

DNA microinjections. For mosaic labeling, zebrafish embryos were collected and injected with appropriate DNA constructs at the 1-4 cell stage. Embryos were injected with $1 \mathrm{nl}$ of a solution containing 15-25 $\mathrm{ng} / \mu \mathrm{l}$ total plasmid DNA, $20 \mathrm{ng} / \mu \mathrm{l}$ transposase mRNA, and $10 \%$ phenol red.

Experimental design and statistical analysis. All experiments were completed with at least three biological replicates, and analyses were completed in a blinded manner. For pharmacological treatments of zebrafish larvae, embryos were treated in groups of 20 embryos. For image analysis, control groups were measured across samples to ensure no differences occurred between controls of different experiments, and then all data were combined for analysis by unpaired $t$ test.

For quantification of cell counts in WT and AKT-DD corpus callosum, 3 individual animals of either sex were killed and fixed as described above. Sections were stained simultaneously and imaged on the same day. Three sections from each animal were imaged, quantified, and averaged per animal. Unpaired $t$ tests on individual animals were completed.

For protein quantification, 3 individual animals were used per sample for mouse samples. For cell culture experiments, experiments were repeated on at least 3 separate days, and samples were collected as described above. Data were analyzed using ANOVA with Dunnett's multiple comparisons test.

For mosaic analysis of myelin production in the zebrafish spinal cord, 1 or 2 cell embryos were injected with pMBP:mEGFP alone or with a second plasmid to manipulate PAK1 function. Larvae were screened for fluorescence at $4 \mathrm{dpf}$, and imaged as described above. Each 
A

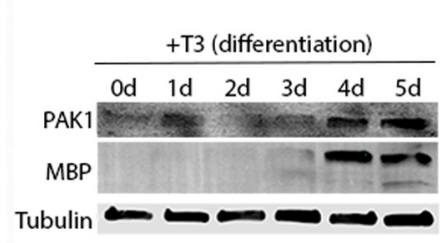

B
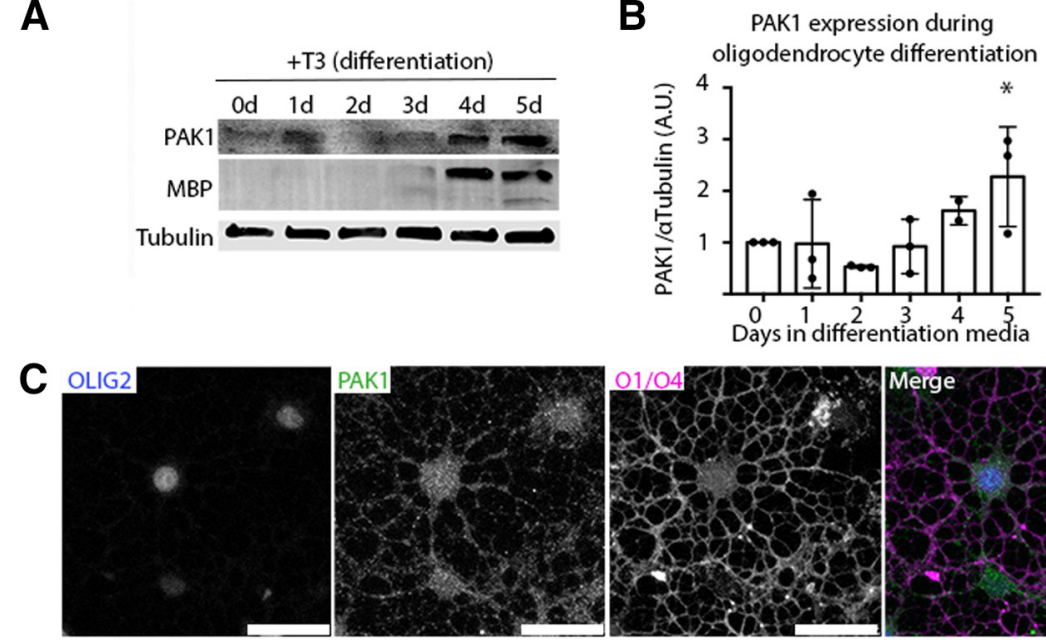

D

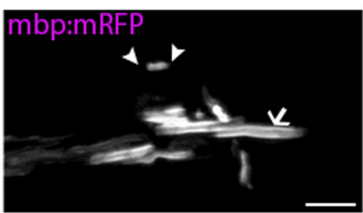

E

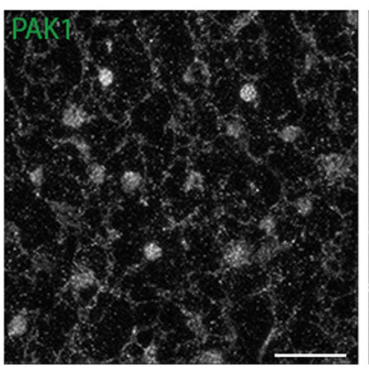

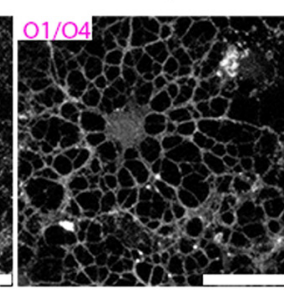
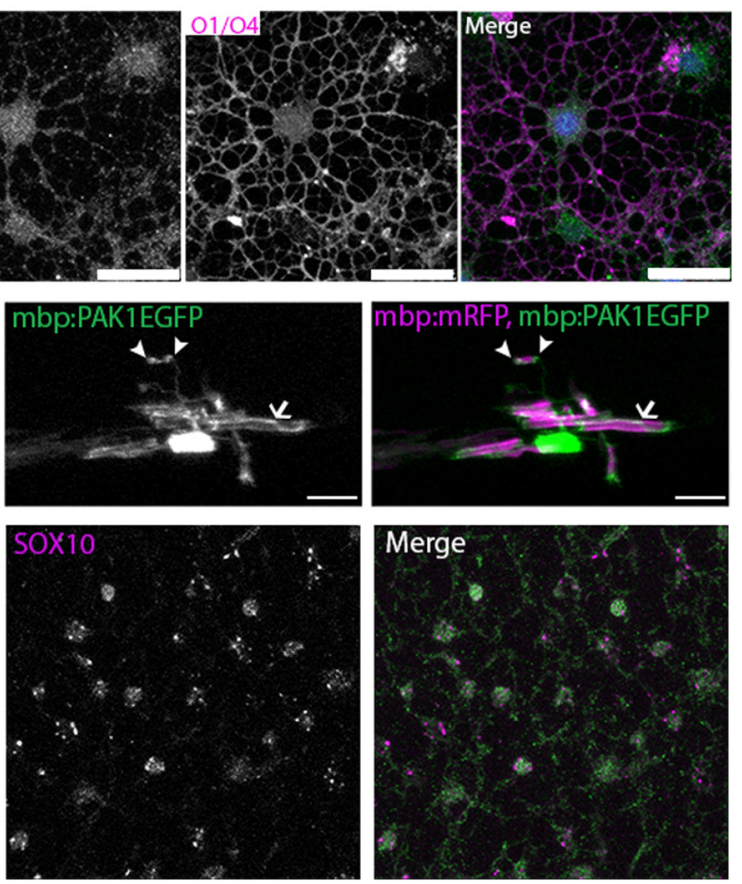

Figure 1. PAK1 expression through the oligodendrocyte lineage. $A$, PAK1 protein expression in vitro in primary rOPCS. PAK1 has increased expression during oligodendrocyte differentiation, particularly when MBP is expressed. $\boldsymbol{B}$, Quantification of in vitro expression of PAK1 protein $\left(F_{(5,17)}=5.583, * p=0.0032\right)$; ordinary one-way ANOVA with Dunnett's multiple comparisons test. $\boldsymbol{C}$, Representative image of PAK1 expression in oligodendrocytes after $5 \mathrm{~d}$ in differentiation media. Green represents PAK1. Blue represents 0lig2. Magenta represents 01/04. Scale bar, $10 \mu \mathrm{m}$. $\boldsymbol{D}, \boldsymbol{E}$, In vivo expression of PAK1 by pEXPRmbp:PAK1-EGFP coexpressed with pEXPR:mbp:mRFP to label myelin. In zebrafish larvae (4 dpf), PAK1 is expressed in the cell body, in presumptive paranodes (arrowheads), and within myelin (arrow). In P8 cortex, PAK1 protein is expressed in oligodendrocytes $(\boldsymbol{E})$ (green represents PAK1; magenta represents SOX10), and P8 brains were fixed in methanol (see Materials and Methods). Scale bar, $25 \mu \mathrm{m}$.

PAK1 manipulation was injected on at least 3 separate days and analyzed using IMARIS 3D software as described above. Controls from each experiment were compared across each experiment. Since no differences were found between controls of individual experiments, internode length and number were averaged per cell, and all individual cells were grouped for analysis using ordinary one-way ANOVA with Dunnett's multiple comparisons test.

\section{Results}

PAK1 expression in oligodendrocytes

PAK1 is highly expressed by multiple cell types in the brain (Kelly and Chernoff, 2012). To investigate PAK1 expression specifically in the oligodendrocyte lineage, we studied primary rat oligodendrocyte differentiation to determine PAK1 protein expression throughout oligodendrocyte development. PAK1 is expressed throughout the oligodendrocyte lineage with highest expression in differentiated oligodendrocytes that express MBP (Fig. 1A,B). This increase in PAK1 protein expression is consistent with PAK1 mRNA levels during OPC differentiation (Y. Zhang et al., 2014; Marques et al., 2016). This suggests that both
PAK1 mRNA and protein are expressed throughout the entire oligodendrocyte lineage but with increasing expression during OPC differentiation and highest expression in myelinating oligodendrocytes ( $\mathrm{Y}$. Zhang et al., 2014; Marques et al., 2016). By immunocytochemistry of cultured cells in vitro, PAK1 protein localizes to both the cell body and the highly branched oligodendrocyte processes in differentiated oligodendrocytes expressing the differentiation surface antigens, $\mathrm{O} 1 / \mathrm{O} 4$ (Fig. 1C).

To assess whether PAK1 is expressed in oligodendrocytes and myelin in vivo, we first performed immunohistochemistry in P8 mouse brains. PAK1 protein localizes to both the oligodendrocyte cell body and oligodendrocyte processes, confirming the expression observed in cells in vitro (Fig. $1 E$ ). To determine whether PAK1 is expressed in myelin, we transiently expressed a full-length PAK1 with an EGFP tag in myelinating zebrafish (pEXPR:mbp:PAK1-EGFP) along with a myelin marker (pEXPR: mbp:mRFP). During active myelination at $4 \mathrm{dpf}$, we observed PAK1 expression in the cell body, presumptive paranodes, and to a lesser extent within the developing myelin internode itself (Fig. 1D). Together with the in vitro expression that shows PAK1 expressed in the cell body and oligodendrocyte branches, this suggests that PAK1 may regulate multiple stages of oligodendrocyte development.

\section{Increasing myelination increases PAK1 expression}

Since PAK1 increases expression during oligodendrocyte differentiation and is most highly expressed in myelinating oligodendrocytes, we tested the hypothesis that increasing myelination would increase PAK1 expression. Our hypermyelinating mouse model driven by constitutively active AKT in oligodendrocytes (PLP-Akt-DD) (Flores et al., 2008; Narayanan et al., 2009) shows increased myelin thickness in the CNS without alterations to the number of differentiated oligodendrocytes, quantified by the number of $\mathrm{CC}^{+}$cells in the corpus callosum (Fig. 2E). These mice continue to actively myelinate as adults. Our data demonstrate that PAK1 expression is increased fourfold in cerebellum of PLP$A k t-D D$ mice, which have increased Akt expression (Fig. $2 A$, $B)$, coincident with the increased myelin wrapping in adult mice (Flores et al., 2008). To confirm that this resulted from increased PAK1 specifically in oligodendrocytes, we analyzed WT and PLP-Akt-DD corpus callosum samples by immunohistochemistry, staining for PAK1 plus the transcription factor SOX10 to identify all oligodendrocyte lineage cells and $\mathrm{CC} 1$ to identify differentiated oligodendrocytes. Immunohistochemical analysis of PLP-Akt-DD corpus callosum indicated a clear increase in PAK1 expression in differentiated 
A

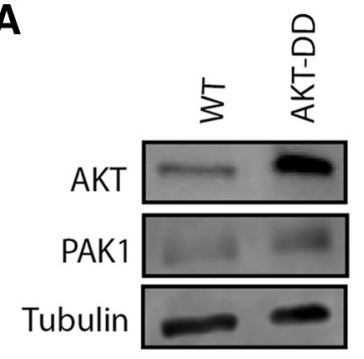

B

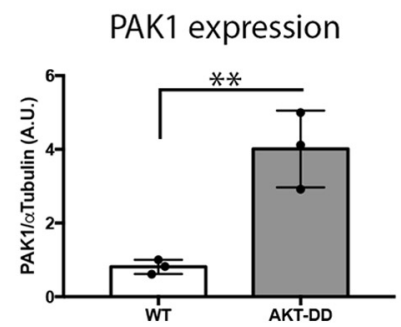

C
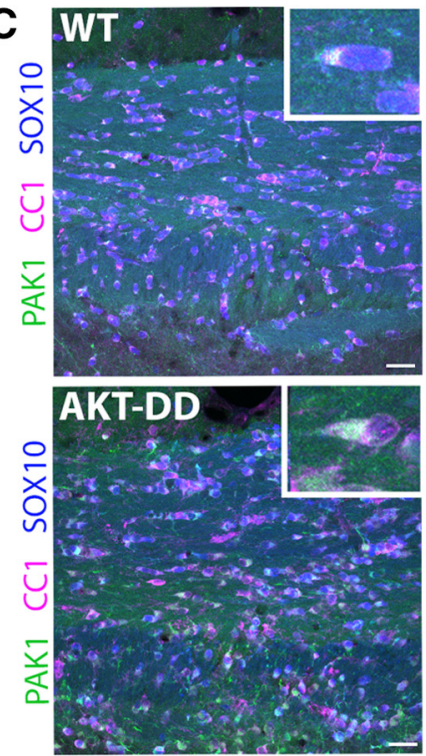

D

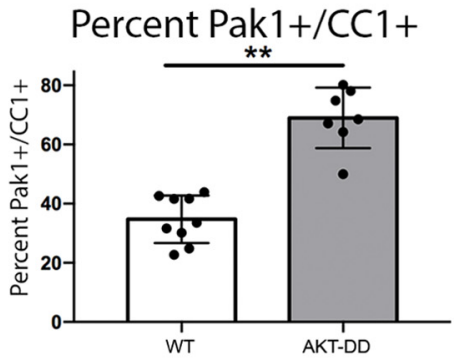

E

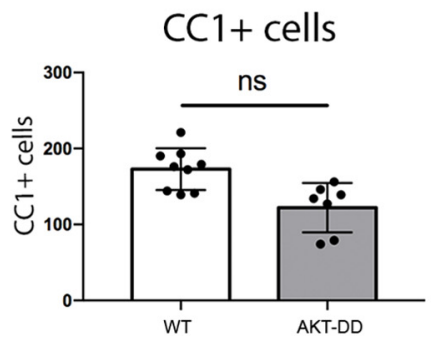

Figure 2. Constitutively active AKT increases PAK1 protein expression in oligodendrocytes. $A$, Representative image of Western blot of WT or AKT-DD cerebellar protein samples, where AKT is overexpressed in PLP-Akt1 mice. B, Quantification of PAK1 protein expression compared with tubulin in WT and AKT-DD cerebellum samples of adult mice. $n=3$ animals/genotype. $t=$ $5.225, \mathrm{df}=4 . * * p=0.0064$ (unpaired $t$ test). C, PAK1 is increased in oligodendrocytes in AKT-DD adult mice in the corpus callosum. Green represents PAK1. Blue represents S0X10 (entire oligodendrocyte lineage). Red represents $\left(C 1\right.$ (differentiated oligodendrocytes). Scale bar, $25 \mu \mathrm{m} . \mathbf{D}, E$, Quantification of the percent of PAK $1^{+} / \mathrm{CC} 1^{+}$cells and of total $\mathrm{CC} 1^{+}$cells. $n=3$ animal/ genotype, 2 or 3 images per animal. $t=8.467, \mathrm{df}=4$. $* * p=0.0011$.

oligodendrocytes, and specifically in the percentage of $\mathrm{PAK}^{+} / \mathrm{CC}^{+}$cells (Fig. $2 C, E$ ). This suggests that PAK1 is downstream of AKT signaling that drives myelination in oligodendrocytes.

\section{Inhibiting Group I PAKs inhibits PAK activity and decreases} myelin gene expression

In order to investigate when during development PAK1 activity was most crucial, we analyzed the impact of PAK1 on oligodendrocyte gene expression during development in zebrafish. Initial studies used PAK1 pharmacological inhibitors; and before studying them in zebrafish, their specificity was established in vitro using the C6 glioma cell line. We stimulated PAK1 activity in the cells with insulin growth factor and tested three different individual chemical inhibitors. Each inhibitor primarily targets PAK1 but also slightly decreases activity of PAK2 and PAK3 (Dolan et al., 2013; Karpov et al., 2015; Ndubaku et al., 2015). Since Group I PAK activity requires autophosphorylation on activity, we quantified PAK activation by $\mathrm{PPAK} 1^{\text {Ser141 }} / \mathrm{PAK} 2^{\text {Ser141 }}$ (Chong et al., 2001). Inhibiting Group I PAKs with each of the three inhibitors decreased PAK activity significantly in C6 cells (Fig. $3 A, B$ ). We then used these inhibitors in our in vivo zebrafish model to investigate the role of PAKs within the oligodendrocyte lineage. The same three chemical inhibitors that inhibited PAK activity in vitro were tested for their impact on myelin gene expression in zebrafish embryos. Inhibiting Group I PAKs from 2 to $4 \mathrm{dpf}$, during the time of oligodendrocyte differentiation and initiation of myelination, led to significantly decreased myelin gene expression. FRAX486 was most effective in decreasing myelin gene expression. Thus, three major zebrafish CNS myelin mRNAs, myelin basic proteins ( $m b p)$, myelin protein zero ( $m p z)$ (Bai et al., 2011), and 36K (Morris et al., 2004), were downregulated after treatment with FRAX486, although only $m b p$ and $36 \mathrm{~K}$ downregulation was statistically significant (Fig. 3C). Thus, Group I PAK activity is needed for normal myelin gene expression during oligodendrocyte differentiation in zebrafish.

\section{PAK1 regulates oligodendrocyte morphology and actin at} oligodendrocyte branch tips

To determine whether Group I PAKs regulate oligodendrocyte morphology in vivo, we inhibited Group I PAKs using the most effective chemical inhibitor (FRAX486). In a transgenic zebrafish line that labels the membranes of oligodendrocytes, $\operatorname{Tg}(n k \times 2.2 \alpha$ : $m E G F P)$, we imaged a lateral view of the spinal cord, and found that PAK1-3 inhibition with FRAX486 from 48 to $56 \mathrm{hpf}$, just before the onset of myelination, reduced oligodendrocyte morphologic complexity. Specifically, the total process length per cell and the number of oligodendrocyte processes (terminal points) were decreased (Fig. 4A-D). We also measured branching complexity by Sholl analysis. Inhibiting PAK1-3 led to a decrease in branching complexity during oligodendrocyte differentiation (Fig. 4C-E). Thus, Group I PAKs positively regulate oligodendrocyte morphology.

Since PAKs are well-known regulators of the actin cytoskeleton (Arias-Romero and Chernoff, 2008) and oligodendrocyte morphologic changes require dramatic changes to the actin cytoskeleton (Nawaz et al., 2015; Zuchero et al., 2015), we next investigated whether PAK regulation of oligodendrocyte differentiation also altered F-actin during this process. To investigate the mechanism of PAK regulation of oligodendrocyte differentiation and process extension, we returned to primary mouse oligodendrocytes and pharmacologically inhibited Group I PAK function in vitro. As oligodendrocytes extend processes in vitro, they develop an actin-rich lamellipodial-like protrusion, or fan, at the tip of individual branches that extends back to the cell body as the branches expand during initial sheet formation. During sheet expansion, F-actin becomes restricted to the outer perimeter of the sheet and is low in the sheet itself. Inhibiting Group I PAKs for $30 \mathrm{~min}$ at $2 \mathrm{~d}$ in OPC media dramatically reduced the number of actin-rich fans in oligodendrocytes, suggesting that PAKs regulate oligodendrocyte morphology through the actin cytoskeleton (Fig. $4 F, G$ ). 


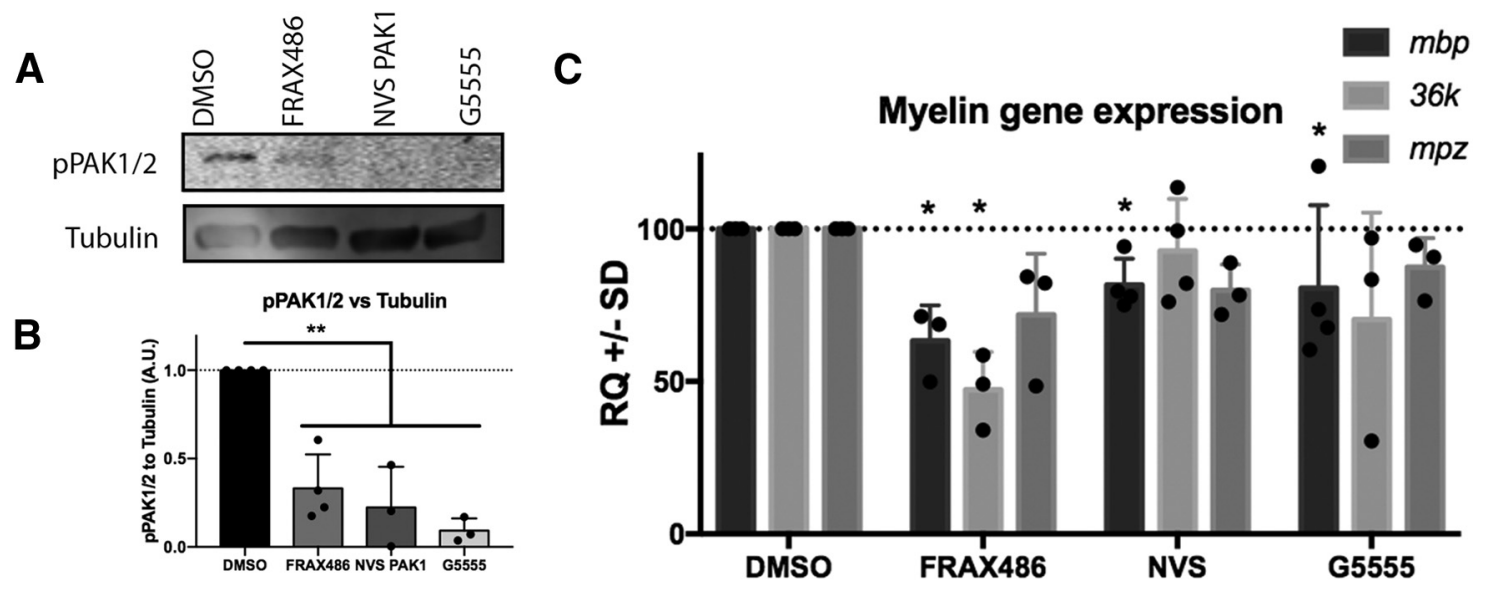

Figure 3. Pharmacologically inhibiting PAK1 activity decreases myelin gene expression. $A$, pPAK1/2 is decreased in 66 cells after 30 min of treatment with PAK inhibitors. $B$, Quantification of pPAK1/2 expression after addition of PAK inhibitors. $n=3$ experiments. $* * p=0.0031$ (ANOVA with Dunnett's multiple comparisons test). C, Myelin mRNAS (mbp, 36k, and mpz) were quantified after treatment of zebrafish from 2 to $4 \mathrm{dpf}$ with DMSO vehicle control or PAK inhibitors, FRAX486, NVS PAK1 1, or G5555 in EM. Each zebrafish sample was a group of 20 fish/treatment. Drugs were refreshed daily. Mbp: $F_{(3,10)}=2.478 ; 36 k: F_{(3,9)}=4.403 ; m p z: F_{(3,9)}=2.539 . * p<0.05 . n=3-5$ biological replicates/treatment.

Inhibiting PAK1 in decreases oligodendrocyte morphology To focus specifically on PAK1, PAK1 shRNAs were transfected into rOPCs to reduce PAK1 expression in vitro, as rOPCs are more reliable for transfection than mouse OPCs. This experiment demonstrated that PAK1 activity had a major impact on oligodendrocyte development by inhibiting process extension. Three different PAK1 shRNA targets and a scrambled control were designed to knock down PAK1 in rOPCs. PAK1 knockdown was confirmed by Western blot in C6 glioma cells, which have higher transfection efficiency than rOPCs. Target 1 and Target 3 showed significant decreases in PAK1 protein level in C6 cells (Fig. 5B), and importantly it also decreased phosphorylation of the downstream pLIMK in these cells, indicating that these shRNAs target PAK1 (Fig. 5A,B). Targets 1 and 3 also decreased PAK1 protein expression in transfected cells (Fig. $5 D$ ).

We transfected rOPCs with the control or PAK1 shRNA (Target 1 and Target 3 ) along with a GFP plasmid. After $4 \mathrm{~h}$, we added fresh differentiation media and allowed the cells to grow for $3 \mathrm{~d}$ (Fig. 5C). Oligodendrocyte morphologic complexity was then quantified in control and PAK1 knockdown cells $\left(\mathrm{GFP}^{+}\right.$ cells). GFP filled cells sufficiently to label major oligodendrocytes processes and quantify gross changes in cell morphologic complexity with static imaging. In PAK1 knockdown cells, oligodendrocyte branching was decreased, compared with the scrambled control (Fig. 6), suggesting that PAK1 normally promotes oligodendrocyte process extension and morphologic complexity.

\section{PAK1 also regulates myelination}

To test the hypothesis that Group I PAKs positively regulate early myelination, we treated zebrafish with the PAK inhibitor FRAX486 during active myelination and determined that Group I PAKs positively regulate myelin internode length. Zebrafish embryos were exposed to FRAX486 for $24 \mathrm{~h}$ (48-72 hpf), during the time when oligodendrocytes are differentiating and beginning to myelinate axons. A lateral view of the spinal cord was then imaged, and the myelin produced was quantified. Since PAK1 can regulate cell migration (Pan et al., 2015), we first assessed the impact of the inhibitor on the number of dorsally migrated OPCs. However, FRAX486 treatment induced no change in the number of dorsally migrated SOX $10^{+}$OPCs (Fig. 7C). When myelin production was then analyzed, Group I PAK inhibition surprisingly resulted in a slight increase in the total number of internodes in the spinal cord (Fig. 7D). Since Group I PAK inhibition led to a decrease in oligodendrocyte terminal points (Fig. 4), the increase in the number of internodes may be because of altered stabilization of initial myelin sheaths after Group I PAK inhibition. Alternatively, it is possible that inhibition of Group I PAK alters PAK signaling in other cell types, such as neurons, that were not specifically assessed here. More importantly, there was a clear reduction in the internode length, an important measure of myelin production (Fig. 7E). This suggests that PAK1-3 positively regulates myelination without altering cell migration.

\section{Oligodendrocyte-specific PAK1 activity regulates myelination}

The data from pharmacological inhibition of Group I PAKs suggested that PAK1-3 positively regulate oligodendrocyte process extension and myelination. Since PAK1 is expressed in multiple cell types (Arias-Romero and Chernoff, 2008); we next altered PAK1 activity specifically in oligodendrocytes, focusing on the actively myelinating cells. PAK1 dimerizes with another PAK1 molecule by the AID binding to the kinase domain of the second PAK1 (Fig. 8A). When GTPase binds to the PAK1 GTPase binding domain, this results in conformational changes, autophosphorylation at multiple sites, including T423, and release of the double-inactive PAK1 forms as activated PAK1 molecules (Fig. $8 B$ ) (Lei et al., 2000). This activation process provides a mechanism to inhibit PAK1 activation by ectopically expressing the AID in cells, where it will act as a dominant-negative molecule by binding to the kinase domain of PAK1 and preventing its activation (Ye and Field, 2012).

To determine whether oligodendrocyte PAK1 activity regulates myelin production, we used the PAK1 AID to inhibit PAK1 function in zebrafish oligodendrocytes after cloning the PAK1 AID behind an oligodendrocyte promoter expressed during active myelination (pEXPR-myrf:PAK1 AID-2AnlsRFP). The 2A peptide is cleavable, which allows expression of the PAK1 AID separate from the red RFP fluorophore, thereby identifying cells expressing the PAK1 AID without directly tagging the protein. Mosaically labeled oligodendrocytes were generated by coinjecting the myrf promoter-driven PAK1 AID plasmid with pEXPRmbp:mEGFP, which labels myelin membrane. Because of the dispersion of individually labeled cells in these mosaic larvae, 
A DMSO Tg(nkx2.2a:mEGFP; sox10:RFP)

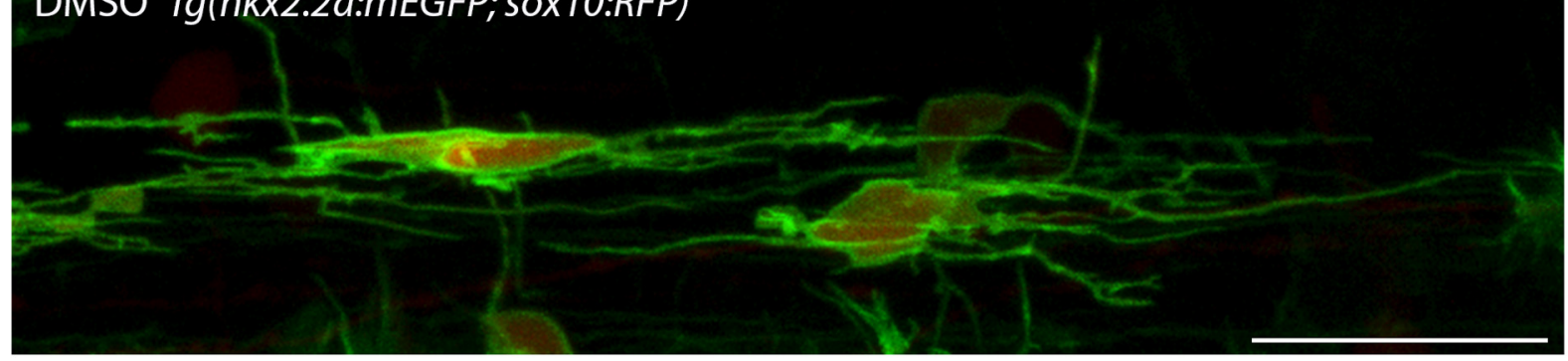

B FRAX486 Tg(nkx2.2a:mEGFP; sox10:RFP)

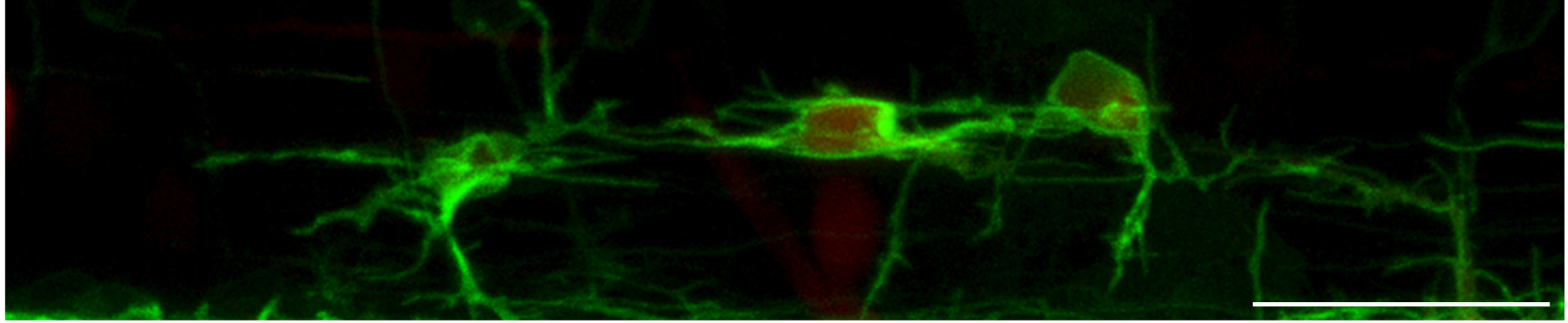

C

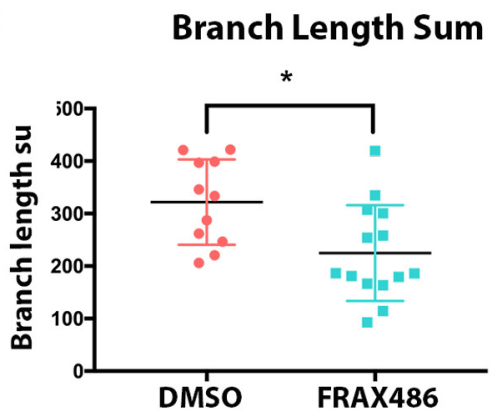

$\mathbf{F}$

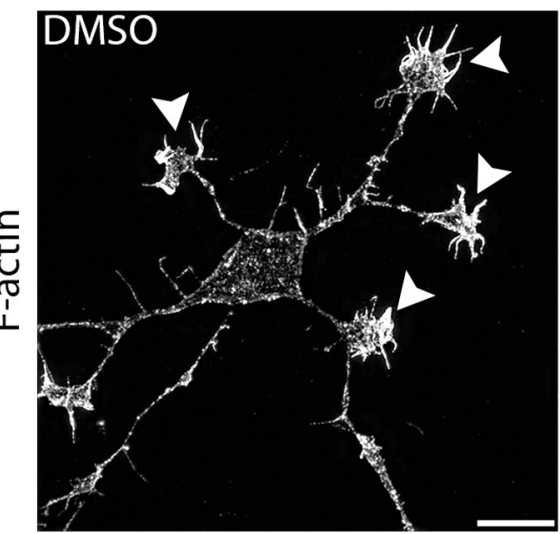

D

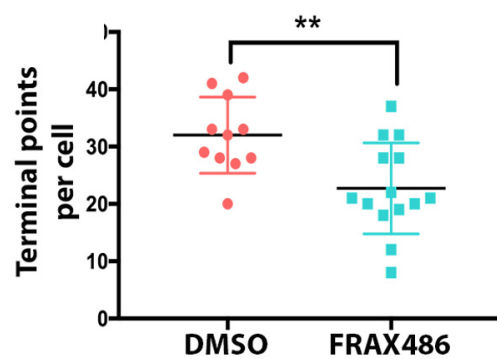

E

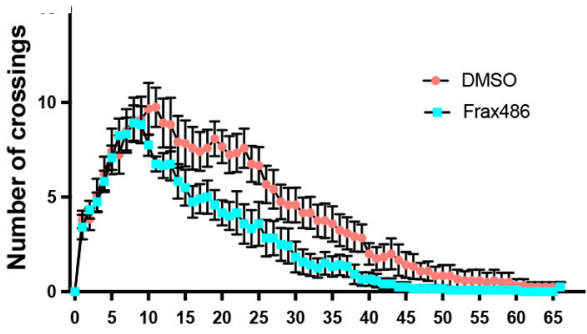

G

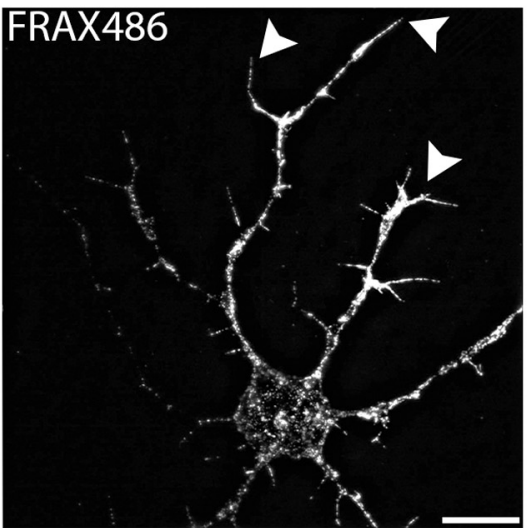

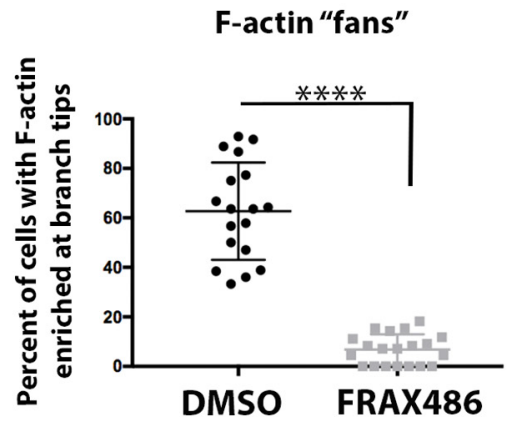

Figure 4. Pharmacologically inhibiting PAK1 activity decreases oligodendrocyte branching complexity and reduces actin in OPC branches. A, B, Tg(nkx2.2:mEGFP; sox10:tagRFP) embryos were treated with a DMS0 vehicle control $(\boldsymbol{A})$ or the PAK1 inhibitor FRAX486 $(\boldsymbol{B})$ from 48 to $56 \mathrm{hpf}$. Images were taken directly above the yolk sac extension. Individual cells were traced and analyzed in 3D using IMARIS software. Scale bar, $25 \mu \mathrm{m}$. C, The inhibition of PAK1 decreased the total length of oligodendrocyte branches. $t=2.771$, $\mathrm{df}=23 . * p=0.01$ (unpaired $t$ test). $\boldsymbol{D}$, The inhibition of PAK1 decreased the number of oligodendrocyte terminal points per cell. $t=3.11, \mathrm{df}=23$. $* * p=0.0049$ (unpaired $t$ test). $\boldsymbol{E}$, Sholl analysis was completed using IMARIS software. $\boldsymbol{F}$, Mouse OPCs were treated for 30 min with DMSO vehicle control or the PAK inhibitor FRAX486 after $2 \mathrm{~d}$ in OPC media. Cells were fixed and stained for F-actin with phalloidin. Representative images at $100 \times$ are shown. Scale bar, $10 \mu \mathrm{m}$. Arrowheads point to the ends of OPC processes. There is dramatic loss of fan-like protrusions after FRAX486 treatment. $N=3$ biological replicates with 5-7 technical replicates per group. $\mathbf{G}$, Quantification of the percent of cells with enriched F-actin in fan-like protrusions at the ends of cell processes (phalloidin stain). Images were taken at $63 \times$, and the number of 0 lig2 ${ }^{+}$cells with F-actin enriched in fan-like protrusions at the ends of cell processes was quantified. White represents DMSO. Gray represents FRAX486-treated cells. $t=12.09, \mathrm{df}=36 . * * * * p<0.0001$ (unpaired $t$ test). 

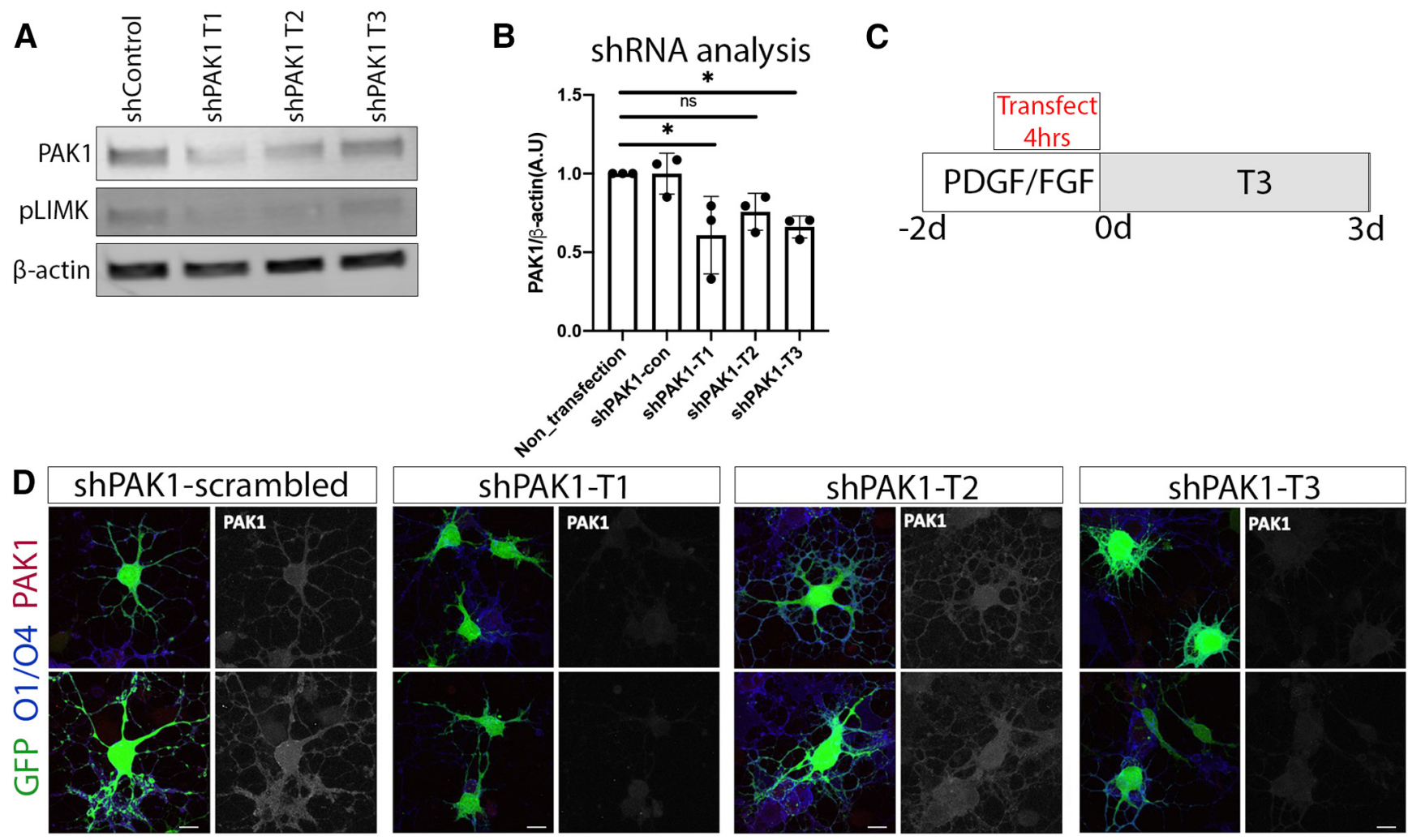

Figure 5. Reducing PAK1 expression decreases pLIMK. $A, B$, Efficacy of PAK1 shRNA was quantified in C 6 cells because of low transfection efficiency of primary rat oligodendrocytes. Thus, PAK1 and its downstream target pLIMK expression was analyzed on Western blot of 6 cells after shRNA transfection. $* p<0.05$ (Dunnett's multiple comparisons test). C, rOPCs were grown in rOPC media with PDGF/FGF for $2 \mathrm{~d}$. Cells were transfected at the last $4 \mathrm{~h}$; then the media was replaced with differentiation media $+\mathrm{T}$, which was replaced every $2 \mathrm{~d}$; and the cells were collected for analysis after $3 \mathrm{~d}$. D, rat OPCs transfected with a scrambled control shRNA (shPAK1-control) or each of the three different PAK1 targets, then differentiated for $3 \mathrm{~d}$. Oligodendrocytes were then collected and stained for GFP to show the impact of shRNA transfection, using 01/04 (blue) as an oligodendrocyte membrane marker and PAK1 (red). Scale bar, $10 \mu \mathrm{m}$.

multiple aspects of myelin production by individual oligodendrocytes in WT or PAK1 $\mathrm{AID}^{+}$cells could be quantified, including changes in myelin internode length or the number of myelin internodes produced by individual oligodendrocytes.

Cell-specific inhibition of PAK1 specifically in oligodendrocytes with the AID construct decreased myelin internode length without altering the number of myelin internodes produced per cell (Fig. 8D,E). In contrast to global inhibition of PAK1-3 observed in our pharmacological manipulations that affects all cell types, this suggests that oligodendrocyte-specific PAK1 positively regulates myelin production through internode length. As a control, the PAK1 AID containing a mutation that prevents its inhibitory function (pEXPR-myrf:PAK1 AID L107F-2AnlsRFP) was injected. Expression of this nonfunctional AID did not alter myelin production (Fig. $8 D$ ). To determine whether the kinase activity is specifically required for myelin production, we inhibited PAK1 activity by expressing a full-length, kinase-dead PAK1 (pEXPR-myrf:PAK1 K299R-2A nlsRFP). This mutant acts as a dominant-negative by binding to endogenous PAK1, preventing kinase activation and downstream signaling (S. Zhang et al., 1995). Inhibiting PAK1 kinase activity with the kinase-dead PAK1 also decreased myelin internode length without changing the number of myelin internodes produced per cell (Fig. 8D,E). Thus, inhibiting PAK1 function specifically in oligodendrocytes by expressing the PAK1 AID or a full-length kinase-dead mutant decreased myelin internode length without altering the number of internodes produced per cell.

In a reciprocal experiment, we constitutively activated PAK1 in oligodendrocytes by overexpressing a constitutively active full-length PAK1 mutant. Mutating threonine 423 to glutamic acid located in the kinase domain of PAK1 increases the autophosphorylation of PAK1 that is required for kinase activation (Zenke et al., 1999). After coinjecting pEXPR-myrf:PAK1 T423E-2AnlsRFP with the myelin marker pEXPR-mbp:mEGFP, myelin internode length and myelin internode number produced per oligodendrocyte were quantified. Activating PAK1 specifically in oligodendrocytes during myelination significantly increased myelin internode length (Fig. 8D,E). Together, these data show that PAK1 regulates myelination through controlling internode length.

\section{Discussion}

The current studies demonstrate that PAK1 promotes oligodendrocyte development and myelin production through internode formation. PAK1 is expressed throughout the oligodendrocyte lineage and most highly expressed in differentiated oligodendrocytes, both in premyelinating and myelinating oligodendrocytes. This expression pattern mimics PAK1 RNA expression, which also shows highest expression in myelinating oligodendrocytes (Y. Zhang et al., 2014; Marques et al., 2016). Despite its higher expression in more mature oligodendrocytes, PAK1 is required for oligodendrocyte morphologic change, including the extensive process extension and retraction during oligodendrocyte differentiation, as well as during active myelination (Fig. 7A). Through global inhibition of Group I PAKs, we demonstrate that PAK1-3 regulates oligodendrocyte morphology by altering the actin cytoskeleton in premyelinating cells, promotes myelin gene 
A
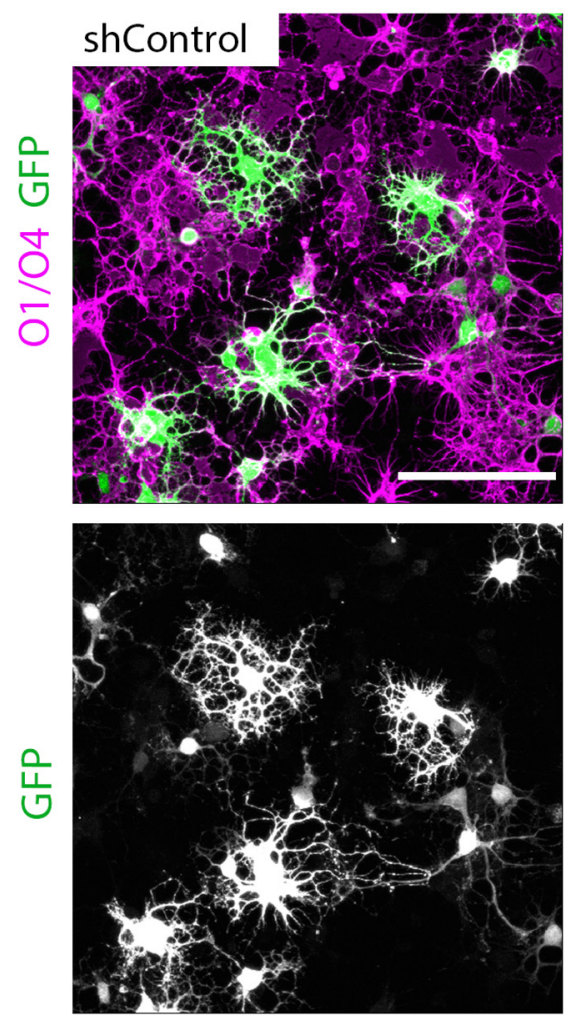

B

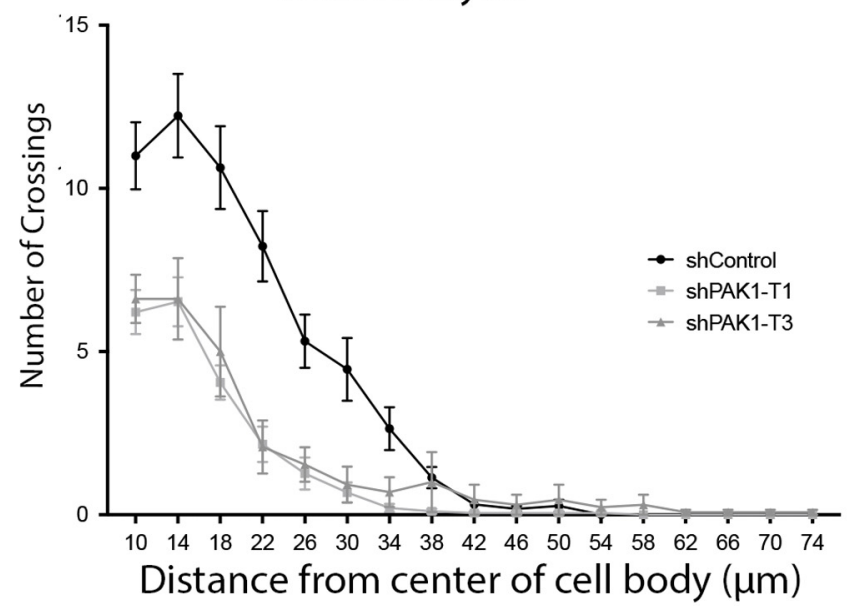

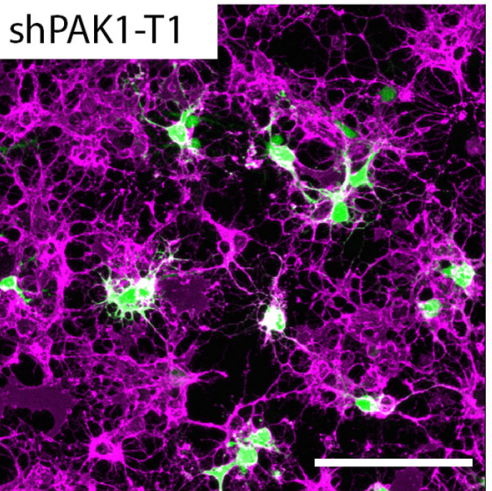
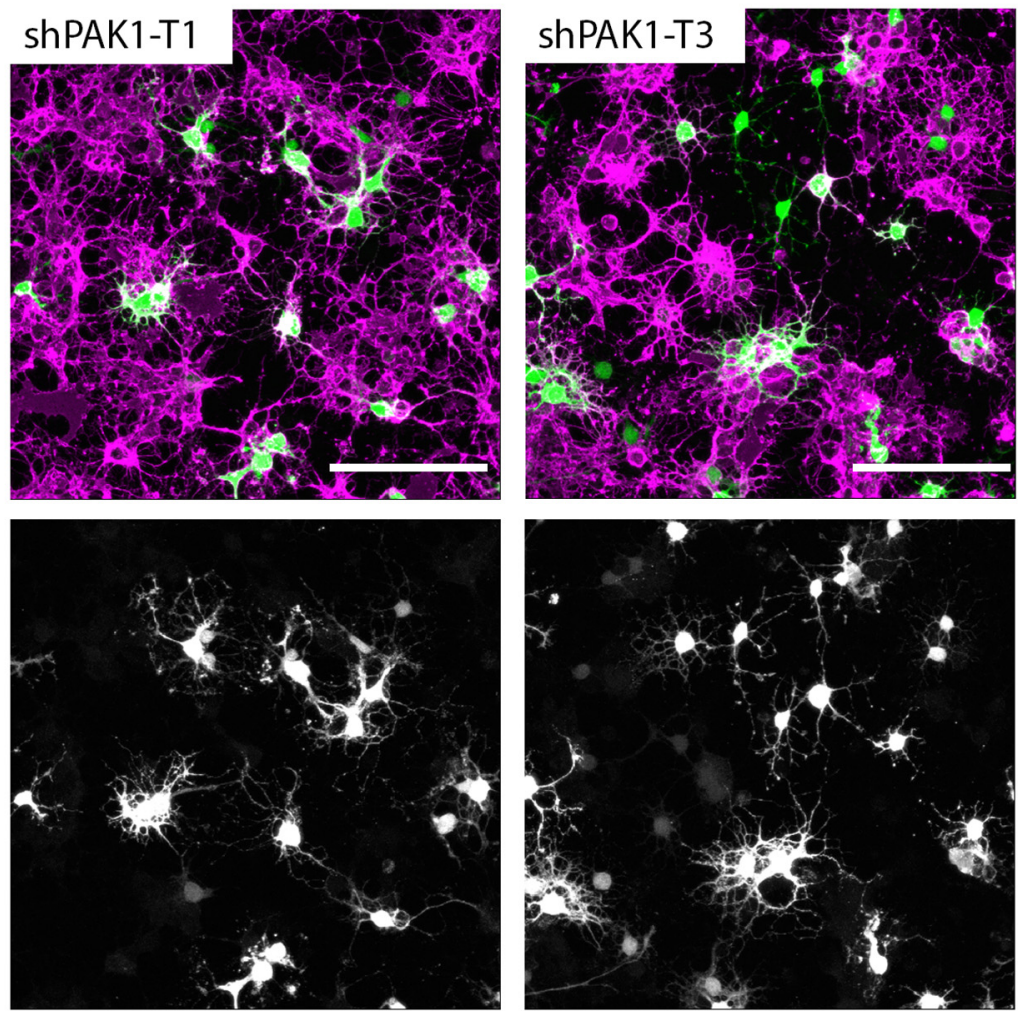

C

Sholl Analysis:

Area under curve

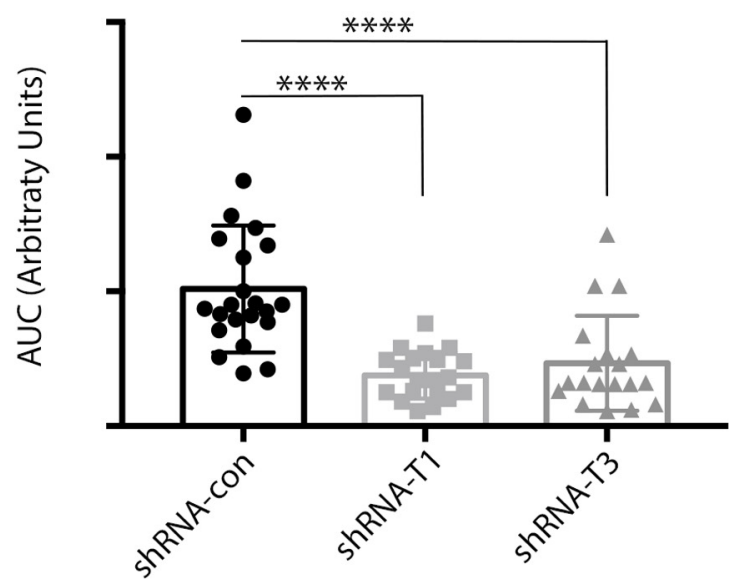

Figure 6. Reducing PAK1 expression decreases oligodendrocyte complexity. A, rOPCs transfected with a scrambled control shRNA (shPAK1-control), or each of the 3 different PAK1 targets was collected and stained for GFP to show the impact of shRNA transfection, using 01/04 (magenta) as an oligodendrocyte membrane marker. Scale bar, $100 \mu \mathrm{m}$. $\boldsymbol{B}$, Sholl analysis of PAK1 knockdown cells in control and shPAK1-T1 and shPAK1-T3. C, Quantification of Sholl analysis by area under the curve per cell (Kruskal-Wallis statistic $=29) . * * * * p<0.0001$ (Kruskal-Wallis test).

expression, and regulates myelination through internode formation. Oligodendrocyte-specific inhibition of PAK1 shows that PAK1 promotes morphologic change and promotes CNS myelination by altering myelin internode length.

Oligodendrocyte development has been investigated in many contexts, studying both its regulation and impact. Numerous studies focus on how specific signaling pathways and ultimately myelin production are regulated during brain development. OPCs proliferate and migrate in response to PDGF signaling, after which they extend numerous processes to differentiate to highly branched premyelinating cells and then myelinating cells. Myelin production is driven by mTOR and ERK signaling, which are likely interacting pathways during oligodendrocyte development (Dai et al., 2014; Ishii et al., 2019). The production of these highly branched cells and the myelin membrane itself requires extensive cytoskeletal rearrangements, and the current studies identify PAK1 as a potential mediator of actin structure and function during oligodendrocyte development.

The actin cytoskeleton is important during both differentiation and myelination, but it plays different roles at these different 


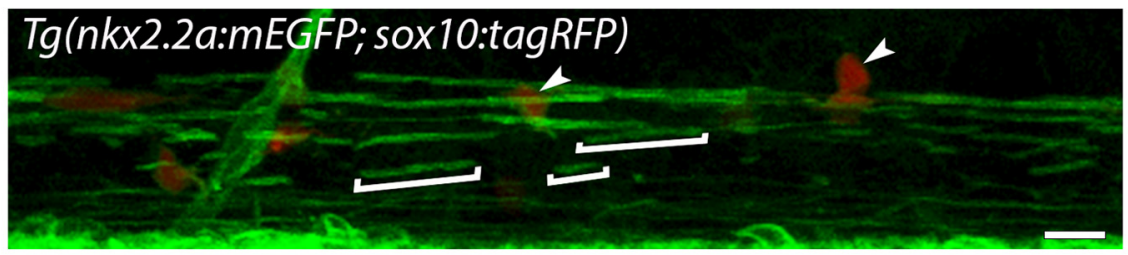

B

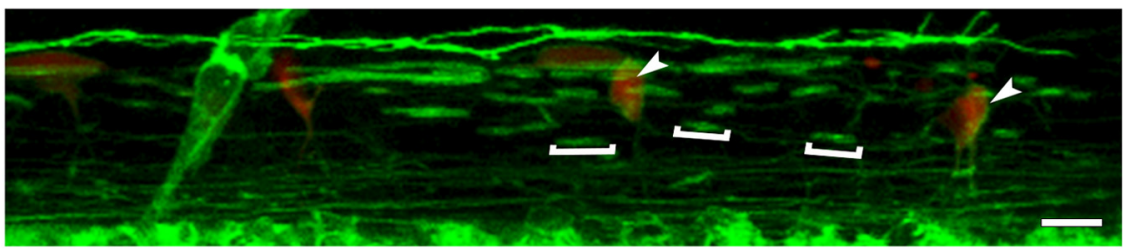

C

$$
\begin{aligned}
& \text { Dorsally } \\
& \text { migrated cells }
\end{aligned}
$$

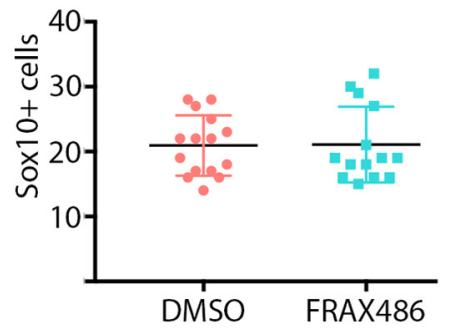

D

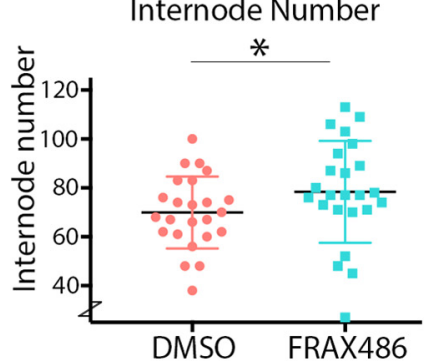

$\mathbf{E}$

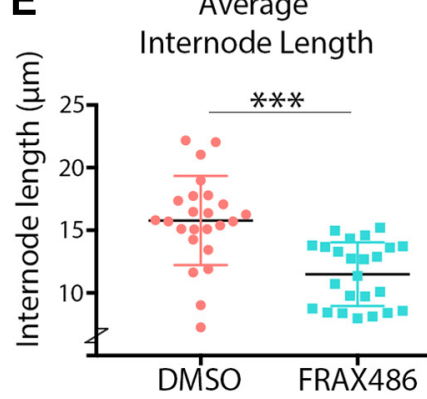

Figure 7. Pharmacologically inhibiting PAK1 activity in vivo alters zebrafish myelin internode length and number. $\boldsymbol{A}, \boldsymbol{B}$, Lateral view of zebrafish spinal cord above yolk sack extension. $T g$ (nkx2.2 2 :mEGFP; sox10:tagRFP) embryos were treated with a DMSO vehicle control $(\boldsymbol{A})$ or the PAK1 inhibitor FRAX486 $(\boldsymbol{B})$ from 48 to 72 hpf, then imaged. Square brackets indicate individual myelin internodes. Arrowheads indicate migrated oligodendrocytes. Scale bar, $50 \mu \mathrm{m}$. C, Quantification of dorsally migrated S0X10 ${ }^{+}$cells labeled by Tg(sox10:tagRFP). D, E, Quantification of myelin internode number $(\boldsymbol{D})$ and length $(\boldsymbol{E})$ represented as the mean/fish. Analysis was completed in IMARIS. $n=3$ experimental replicas, 24 fish/treatment group. Wilcoxon rank-sum, internode number: $r=0.286, * p=0.049$, internode length: $r=0.619, * * * p=1.9 \mathrm{e}-05$.

stages of oligodendrocyte maturation (for review, see Brown and Macklin, 2020). In OPCs, F-actin localizes to branches and is highly expressed at the tips of processes that interact with the local environment (Kim et al., 2006; Nawaz et al., 2015). The tips of these processes are reminiscent of lamellipodia, spreading out like a fan and enriched in the actin cytoskeleton (Ridley, 2011; Nawaz et al., 2015) (Fig. 4). Actin polymerization is required for later oligodendrocyte process extension where processes extend, contact, and ensheath axons in the early stages of myelin wrapping (Nawaz et al., 2015; Zuchero et al., 2015). During active myelin wrapping, it is hypothesized that filamentous actin localizes to the leading edge of the myelin wrap, expanding and moving this inner tongue between the axon and previous myelin wraps (Nawaz et al., 2015; Zuchero et al., 2015). In these models, F-actin is subsequently depolymerized immediately behind the inner tongue, allowing for myelin compaction and lateral spreading (Fig. 9D,E). Additionally, F-actin maintains cytoplasmic channels in an open state within myelin. This allows for movement of myelin proteins and molecular machinery throughout myelin, similar to Schmitt-Lanterman clefts in the PNS (Velumian et al., 2011; Snaidero et al., 2014, 2017). Thus, F-actin dynamics within myelin require precise, tight regulation to control myelin structure and the movement of cytoplasm.

PAK1 is a small molecule that can be quickly activated and inactivated, providing a promising target for regulating these processes. PAK1 activation occurs by multiple mechanisms, but its canonical activation is by GTPases. Upon GTPase binding, PAK1 dimers undergo conformational changes and autophosphorylation, after which the activated PAK1 monomers phosphorylate LIMK. LIMK then phosphorylates the actin depolymerizing factor cofilin, ultimately leading to actin cytoskeletal rearrangement (Fig. 9) (Edwards et al., 1999; Lei et al., 2000). It would be important in future studies to determine whether PAK1 alters actin dynamics within oligodendrocytes.

Importantly, PAK1 can also be activated in a GTPase-independent manner, through multiple signaling molecules. PI-3K can directly induce PAK1 activity, after which PAK1 directly phosphorylates actin, leading to dissolution of stress fibers (Papakonstanti and Stournaras, 2002). AKT can also activate PAK1 in a GTPase-independent manner, increasing cell survival (Tang et al., 2000). Together, these data indicate that PAK1 function depends on the upstream activation signal and that both GTPase activity and direct PI-3K/AKT signaling may regulate its activity. The data presented here demonstrate that increasing AKT increases PAK1 in oligodendrocytes (Fig. 2). Determining whether AKT directly activates PAK1 will be important for future investigations. This is particularly important as AKT signaling can both promote or inhibit myelination. For example, in the PNS, the AKT/mTORC1 pathway regulates the myelination pathway differently depending on the timing of AKT/mTORC1 activation and the activation of specific downstream targets (Figlia et al., 2017). Additionally, the similarity among Group I PAKs suggests that PAK family members may be able to compensate for loss of one family member. Whether upstream signals regulate multiple PAK family members and whether PAK2 or PAK 3 could compensate for loss of PAK1 within the oligodendrocyte lineage will be important future directions.

During oligodendrocyte differentiation, our data suggest that PAK1 drives process extension, and inhibiting PAK1 leads to a decrease in actin-rich fans at the edges of oligodendrocyte processes (Fig. 4). Both cell adhesion molecules and growth factors 
A

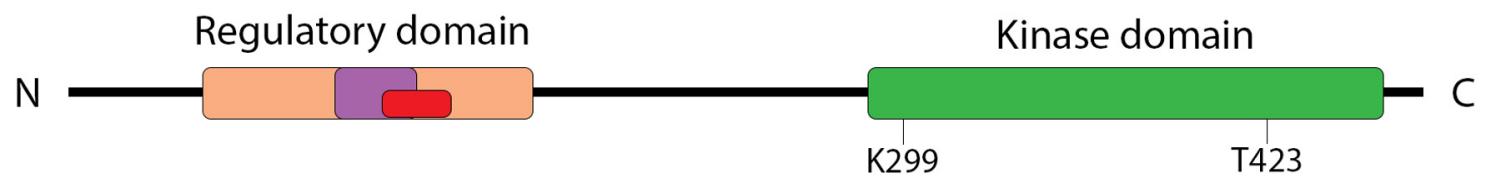

B

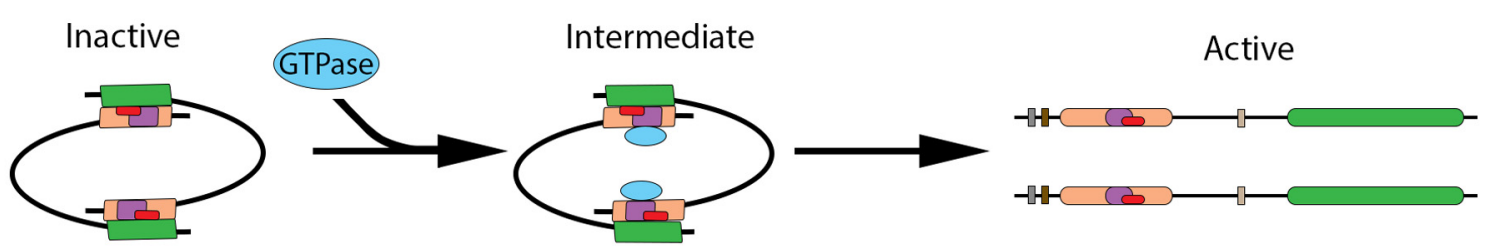

C
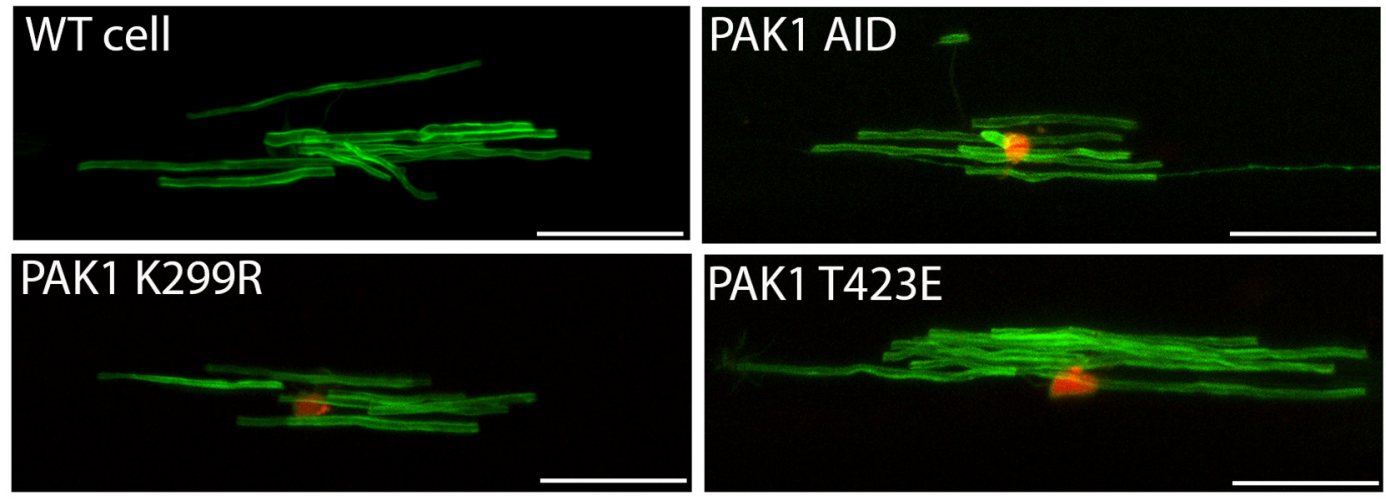

D

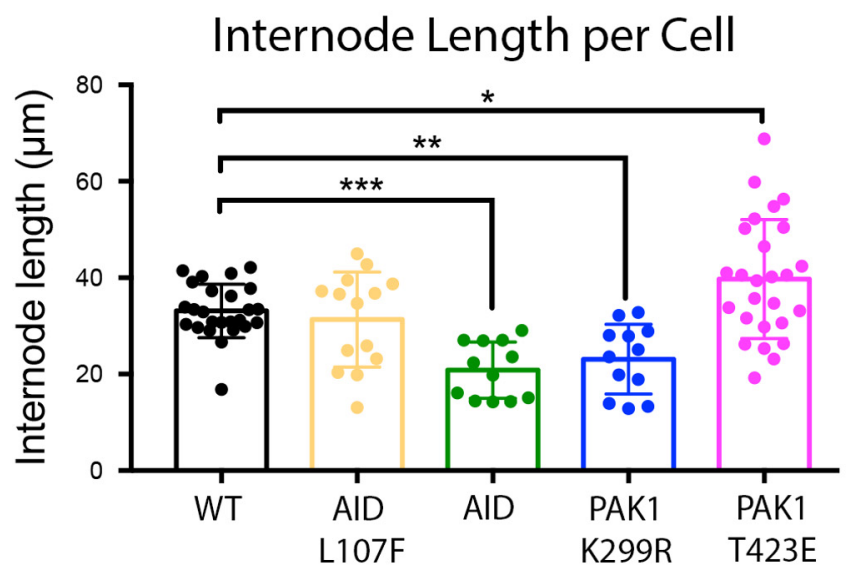

E

Internode Number per Cell

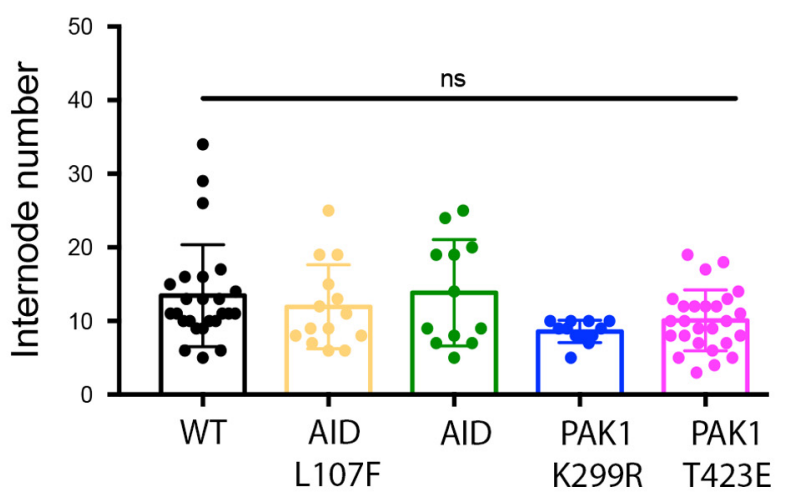

Figure 8. Oligodendrocyte-specific PAK1 regulates myelin internode length. A, Schematic of PAK1 protein. PAK1 contains Nck and Grb2 binding domains at the N-terminus. The regulatory domain contains a GTPase binding domain (GBD) and an overlapping AID. There is a PIX binding domain, and then the kinase domain, which contains multiple phosphorylation sites, including K299 and T423, both of which are important for PAK1 activation. $\boldsymbol{B}$, Inactive PAK1 folds into a homodimer where the AID of one PAK1 binds to the kinase domain of another PAK1, rendering both inactive. GTPase binding to the GBD results in a conformational change, autophosphorylation, and activation of the kinase domain. $C$, Representative images of myelin internodes. Zebrafish embryos were injected with pMBP:mEGFP along with a second plasmid to manipulate PAK1 function. The second plasmids were as follows: PAK1 AID, which blocks PAK1 activation; dominant negative PAK1 (PAK1 K299R), which blocks PAK1 activity; constitutively active PAK1 (PAK1T423E), which maintains PAK1 activity; or AID L107F, which eliminates the AID function, allowing normal PAK1 activation. Scale bar, $25 \mu \mathrm{m}$. D, Quantification of internode length, determined by the mean length of all internodes/cell. $\boldsymbol{E}$, Quantification of internode number. Internode number was determined by the number of myelin internodes produced/cell. Zebrafish images were quantified using IMARIS software. Controls from all experiments were combined, $n=12-26$ cells/group. Ordinary one-way ANOVA with Dunnett's multiple comparisons test $\left(F_{(4,84)}=12.49\right) . * p=0.0356 . * * p=0.0077 . * * * p=0.0008$. 
A

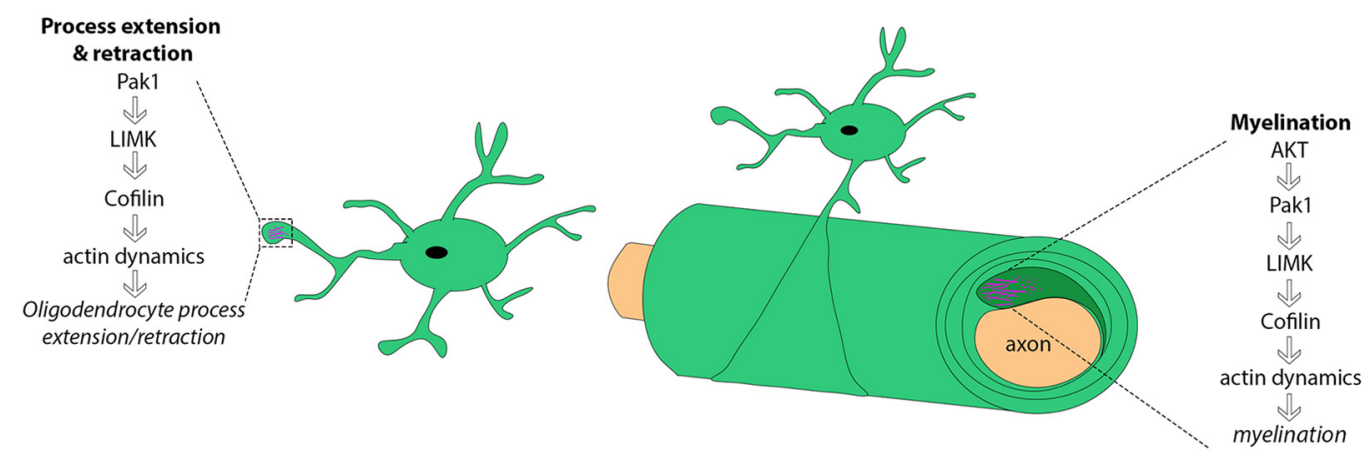

B

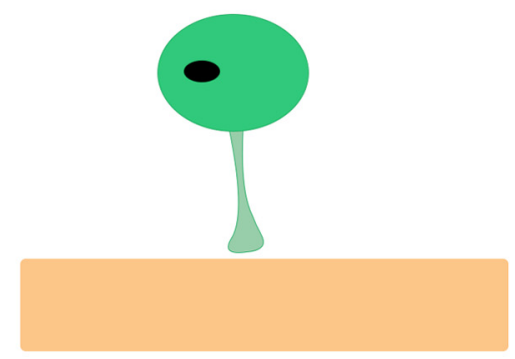

Oligodendrocyte process extension

D

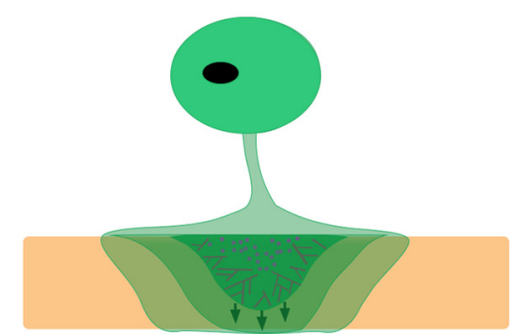

Myelination:

F-actin pushes the inner tongue between previous myelin wraps and the axon

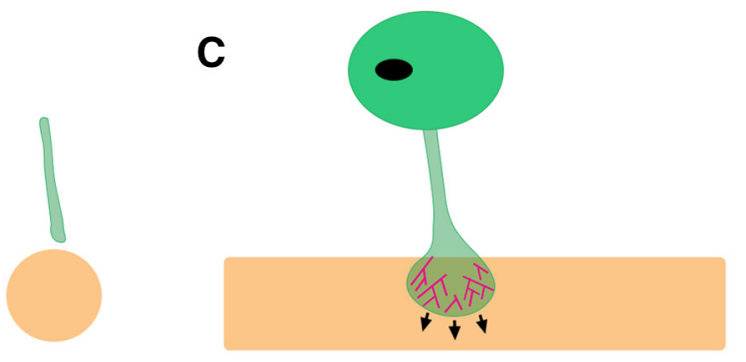

Axon ensheathment

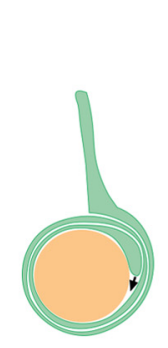

E

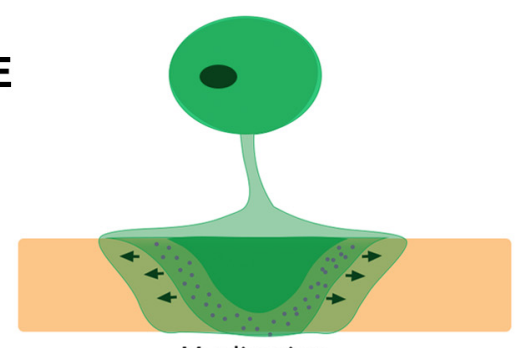

Myelination:

G-actin allows for myelin compaction

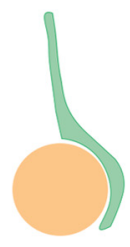

(n)

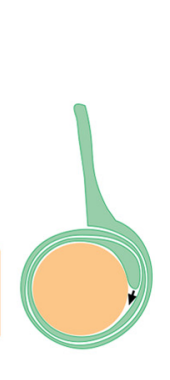

\footnotetext{
which drives lateral extension
}

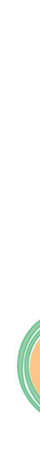

\section{.}

$$
\text { . }
$$


profiles vary along CNS axons. Some axons are continuously myelinated, some have intermittent myelin, while others have no myelin at all (Tomassy et al., 2014). Additionally, parameters, such as myelin thickness, myelin internode length, the paranodal domains, or the size of the nodes of Ranvier, where voltage-gated sodium channels are located, also vary. Thus, unique myelin profiles provide a mechanism to adjust neuronal conduction velocity within neural circuits. Myelin internode length has important implications for neuronal function. In the auditory system, myelin internode length is a major mechanism regulating conduction velocity (Seidl et al., 2010, 2014), which influences the ability to localize sound. Our studies suggest that PAK1 activity may be one of several regulators of myelin internode length in these different contexts.

Given its impact on myelin internode length (Figs. 7, 8) and our zebrafish expression studies (Fig. 1D), it is intriguing to speculate that PAK1 could localize to the inner tongue during myelin wrapping, although myelin wrapping was not specifically investigated in these studies. Since PAK1 can quickly be activated or deactivated, this would provide an opportunity to switch rapidly from actin polymerization to actin depolymerization, which is required to drive myelin wrapping (Fig. 9). Alternatively, PAK1 could modulate signaling pathways, including AKT and ERK1/2, known to promote myelination. Modulating AKT can also alter the actin cytoskeleton in oligodendrocytes, suggesting that AKT and PAK1 could work synergistically to regulate the actin cytoskeleton in oligodendrocytes. For example, inhibiting AKT in vitro decreases the $\mathrm{F}$-actin rim, whereas activating AKT increases the F-actin rim in differentiated oligodendrocytes (Snaidero et al., 2014). It is unclear whether PAK1 activation in oligodendrocytes occurs in a GTPase-dependent manner, and it is intriguing that PAK1 is increased in AKT-overexpressing hypermyelinating mice (Fig. 2). That may result from GTPase-independent direct activation of PAK1 by AKT (Tang et al., 2000) or as a consequence of continual myelination driven by AKT/mTOR in these mice (Flores et al., 2008; Narayanan et al., 2009), which would likely require increased PAK1 activity for increased myelination.

PAK1 activity in myelinating cells is clearly tightly regulated. Our studies suggest that PAK1 may play multiple roles throughout oligodendrocyte differentiation and myelination depending on the timing of activation, as well as its upstream activation. PAK1 is important in PNS myelination, and its activity impacts myelin in a model of hereditary neuropathy with liability of pressure palsies, which has mild myelin dysfunction. These mice have a decrease in compound action potential and an increase in F-actin at the paranodes, cytoplasmic domains of myelin that contact axons. Importantly, they have increased PAK1 activity in myelin, suggesting that overactivating PAK1 can be detrimental for myelination (Hu et al., 2016). Together with our data in the CNS, this suggests that PAK1 activity must be tightly regulated for proper myelination, as either too little or too much PAK1 activity is detrimental for proper myelination.

Data in the PNS demonstrate that AKT function differs depending on the stage of Schwann cell development. Before PNS myelination begins, AKT inhibits Schwann cell differentiation through mTOR and its downstream target S6 kinase, but later, AKT promotes myelin wrapping through both mTOR-dependent and -independent pathways (Figlia et al., 2017). Our studies indicate that PAK1 impacts two stages of oligodendrocyte development. One could speculate that PAK1 might also function through two different mechanisms in oligodendrocytes in the CNS, promoting oligodendrocyte differentiation and myelin wrapping via two independent mechanisms.
In conclusion, we have shown that PAK1 is a positive regulator of oligodendrocyte morphology during differentiation and myelination through internode formation. Exploring the downstream targets of PAK1 in myelinating cells will be important for future investigations. It will also be essential to determine whether oligodendrocyte PAK1 activity responds to neuronal activity or other signals, such as cell adhesion molecules, that interact with the inner tongue during myelination.

\section{References}

Arias-Romero LE, Chernoff J (2008) A tale of two Paks. Biol Cell 100:97-108. Bai Q, Sun M, Stolz DB, Burton EA (2011) Major isoform of zebrafish P0 is a $23.5 \mathrm{kDa}$ myelin glycoprotein expressed in selected white matter tracts of the central nervous system. J Comp Neurol 519:1580-1596.

Bercury KK, Dai J, Sachs HH, Ahrendsen JT, Wood TL, Macklin WB (2014) Conditional ablation of raptor or rictor has differential impact on oligodendrocyte differentiation and CNS myelination. J Neurosci 34:44664480 .

Brown TL, Macklin WB (2020) The actin cytoskeleton in myelinating cells. Neurochem Res 45:684-693.

Bustin SA, Benes V, Garson JA, Hellemans J, Huggett J, Kubista M, Mueller R, Nolan T, Pfaffl MW, Shipley GL, Vandesompele J, Wittwer CT (2009) The MIQE guidelines: minimum information for publication of quantitative real-time PCR experiments. Clin Chem 55:611-622.

Chong C, Tan L, Lim L, Manser E (2001) The mechanism of PAK activation: autophosphorylation events in both regulatory and kinase domains control activity. J Biol Chem 276:17347-17353.

Dai J, Bercury KK, Macklin WB (2014) Interaction of mTOR and Erk1/2 signaling to regulate oligodendrocyte differentiation. Glia 62:2096-2109.

Dai J, Bercury KK, Weilin J, Macklin WB (2015) Olig1 acetylation and nuclear exporr mediate oligodendrocyte development. J Neurosci 35:1587515893.

Dolan BM, Duron SG, Campbell DA, Vollrath B, Shankaranarayana Rao BS, Ko HY, Lin GG, Govindarajan A, Choi SY, Tonegawa S (2013) Rescue of fragile X syndrome phenotypes in Fmr1 KO mice by the small-molecule PAK inhibitor FRAX486. Proc Natl Acad Sci USA 110:5671-5676.

Edwards DC, Sanders LC, Bokoch GM, Gill GN (1999) Activation of LIM-kinase by Pak1 couples Rac/Cdc42 GTPase signalling to actin cytoskeletal dynamics. Nat Cell Biol 1:253-259.

Ellison JA, de Vellis J (1994) Platelet-derived growth factor receptor is expressed by cells in the early oligodendrocyte lineage. J Neurosci Res 37:116-128.

Figlia G, Norrmén C, Pereira JA, Gerber D, Suter U (2017) Dual function of the PI3K-Akt-mTORC1 axis in myelination of the peripheral nervous system. eLife 6:7891.

Flores AI, Narayanan SP, Morse EN, Shick HE, Yin X, Kidd G, Avila RL, Kirschner DA, Macklin WB (2008) Constitutively active Akt induces enhanced myelination in the CNS. J Neurosci 28:7174-7183.

Frost JA, Steen H, Shapiro P, Lewis T, Ahn N, Shaw PE, Cobb MH (1997) Cross-cascade activation of ERKs and ternary complex factors by Rho family proteins. EMBO J 16:6426-6438.

Goebbels S, Oltrogge JH, Kemper R, Heilmann I, Bormuth I, Wolfer S, Wichert SP, Möbius W, Liu X, Lappe-Siefke C, Rossner MJ, Groszer M, Suter U, Frahm J, Boretius S, Nave KA (2010) Elevated phosphatidylinositol 3,4,5-trisphosphate in glia triggers cell-autonomous membrane wrapping and myelination. J Neurosci 30:8953-8964.

Higuchi M, Onishi K, Kikuchi C, Gotoh Y (2008) Scaffolding function of PAK in the PDK1-Akt pathway. Nat Cell Biol 10:1356-1364.

Hines JH, Ravanelli AM, Schwindt R, Scott EK, Appel B (2015) Neuronal activity biases axon selection for myelination in vivo. Nat Neurosci 18:683689.

Hu B, Arpag S, Zhang X, Möbius W, Werner H, Sosinsky G, Ellisman M, Zhang Y, Hamilton A, Chernoff J, Li J (2016) Tuning PAK activity to rescue abnormal myelin permeability in HNPP. PLoS Genet 12:e1006290.

Huang W, Zhou Z, Asrar S, Henkelman M, Xie W, Jia Z (2011) p21-activated kinases 1 and 3 control brain size through coordinating neuronal complexity and synaptic properties. Mol Cell Biol 31:388-403.

Hughes EG, Kang SH, Fukaya M, Bergles DE (2013) Oligodendrocyte progenitors balance growth with self-repulsion to achieve homeostasis in the adult brain. Nat Neurosci 16:668-676. 
Ishii A, Fyffe-Maricich SL, Furusho M, Miller RH, Bansal R (2012) ERK1/ ERK2 MAPK signaling is required to increase myelin thickness independent of oligodendrocyte differentiation and initiation of myelination. J Neurosci 32:8855-8864.

Ishii A, Furusho M, Bansal R (2013) Sustained activation of ERK1/2 MAPK in oligodendrocytes and Schwann cells enhances myelin growth and stimulates oligodendrocyte progenitor expansion. J Neurosci 33:175-186.

Ishii A, Furusho M, Macklin W, Bansal R (2019) Independent and cooperative roles of the Mek/ERK1/2-MAPK and PI3K/Akt/mTOR pathways during developmental myelination and in adulthood. Glia 67:1277-1295.

Karpov AS, Amiri P, Bellamacina C, Bellance MH, Breitenstein W, Daniel D, Denay R, Fabbro D, Fernandez C, Galuba I, Guerro-Lagasse S, Gutmann S, Hinh L, Jahnke W, Klopp J, Lai A, Lindvall MK, Ma S, Möbitz H, Pecchi S, et al. (2015) Optimization of a dibenzodiazepine hit to a potent and selective allosteric PAK1 inhibitor. ACS Med Chem Lett 6:776-781.

Kelly ML, Chernoff J (2012) Mouse models of PAK function. Cell Logist 2:84-88.

Kim HJ, DiBernardo AB, Sloane JA, Rasband MN, Solomon D, Kosaras B, Kwak SP, Vartanian TK (2006) WAVE1 is required for oligodendrocyte morphogenesis and normal CNS myelination. J Neurosci 26:5849-5859.

Kimmel CB, Ballard WW, Kimmel SR, Ullmann B, Schilling TF (1995) Stages of Embryonic Development of the Zebrafish. Dev Dyn 203:253310 .

Kirby BB, Takada N, Latimer AJ, Shin J, Carney TJ, Kelsh RN, Appel B (2006) In vivo time-lapse imaging shows dynamic oligodendrocyte progenitor behavior during zebrafish development. Nat Neurosci 9:15061511.

Kwan KM, Fujimoto E, Grabher C, Mangum BD, Hardy ME, Campbell DS, Parant JM, Yost HJ, Kanki JP, Chien C-B (2007) The Tol2kit: a multisite gateway-based construction kit for Tol2 transposon transgenesis constructs. Dev Dyn 236:3088-3099.

Lee Y, Morrison BM, Li Y, Lengacher S, Farah MH, Hoffman PN, Liu Y, Tsingalia A, Jin L, Zhang PW, Pellerin L, Magistretti PJ, Rothstein JD (2012) Oligodendroglia metabolically support axons and contribute to neurodegeneration. Nature 487:443-448.

Lei M, Lu W, Meng W, Parrini MC, Eck MJ, Mayer BJ, Harrison SC (2000) Structure of PAK1 in an autoinhibited conformation reveals a multistage activation switch. Cell 102:387-397.

Li S, Leshchyns'ka I, Chernyshova Y, Schachner M, Sytnyk V (2013) The neural cell adhesion molecule (NCAM) associates with and signals through p21-activated kinase 1 (Pak1). J Neurosci 33:790-803.

Liang X, Draghi NA, Resh MD (2004) Signaling from integrins to Fyn to Rho family GTPases regulates morphologic differentiation of oligodendrocytes. J Neurosci 24:7140-7149.

Maglorius Renkilaraj MR, Baudouin L, Wells CM, Doulazmi M, Wehrlé R, Cannaya V, Bachelin C, Barnier JV, Jia Z, Nait Oumesmar B, Dusart I, Bouslama-Oueghlani L (2017) The intellectual disability protein PAK3 regulates oligodendrocyte precursor cell differentiation. Neurobiol Dis 98:137-148.

Manser E, Leung T, Salihuddin H, Zhao ZS, Lim L (1994) A brain serine/threonine protein kinase activated by Cdc42 and Racl. Nature 367:40-46.

Marques S, Zeisel A, Codeluppi S, van Bruggen D, Mendanha Falcão A, Xiao L, Li H, Häring M, Hochgerner H, Romanov RA, Gyllborg D, Muñoz Manchado A, La Manno G, Lönnerberg P, Floriddia EM, Rezayee F, Ernfors P, Arenas E, Hjerling-Leffler J, Harkany T, et al. (2016) Oligodendrocyte heterogeneity in the mouse juvenile and adult central nervous system. Science 352:1326-1329.

Mensch S, Baraban M, Almeida R, Czopka T, Ausborn J, El Manira A, Lyons DA (2015) Synaptic vesicle release regulates myelin sheath number of individual oligodendrocytes in vivo. Nat Neurosci 18:628-630.

Morris JK, Willard BB, Yin X, Jeserich G, Kinter M, Trapp BD (2004) The $36 \mathrm{~K}$ protein of zebrafish CNS myelin is a short-chain dehydrogenase. Glia 45:378-391.

Narayanan SP, Flores AI, Wang F, Macklin WB (2009) Akt signals through the mammalian target of rapamycin pathway to regulate CNS myelination. J Neurosci 29:6860-6870.

Nawaz S, Sánchez P, Schmitt S, Snaidero N, Mitkovski M, Velte C, Brückner BR, Alexopoulos I, Czopka T, Jung SY, Rhee JS, Janshoff A, Witke W, Schaap IA, Lyons DA, Simons M (2015) Actin filament turnover drives leading edge growth during myelin sheath formation in the central nervous system. Dev Cell 34:139-151.
Ndubaku CO, Crawford JJ, Drobnick J, Aliagas I, Campbell D, Dong P, Dornan LM, Duron S, Epler J, Gazzard L, Heise CE, Hoeflich KP, Jakubiak D, La H, Lee W, Lin B, Lyssikatos JP, Maksimoska J, Marmorstein R, Murray LJ, et al. (2015) Design of selective PAK1 inhibitor G-5555: improving properties by employing an unorthodox low-pKa polar moiety. ACS Med Chem Lett 6:1241-1246.

O'Meara RW, Ryan SD, Colognato H, Kothary R (2011) Derivation of enriched oligodendrocyte cultures and oligodendrocyte/neuron myelinating co-cultures from post-natal murine tissues. J Vis Exp 54:3324.

Pan X, Chang X, Leung C, Zhou Z, Cao F, Xie W, Jia Z (2015) PAK1 regulates cortical development via promoting neuronal migration and progenitor cell proliferation. Mol Brain 8:36.

Papakonstanti EA, Stournaras C (2002) Association of PI-3 kinase with PAK1 leads to actin phosphorylation and cytoskeletal reorganization. Mol Biol Cell 13:2946-2962.

Ridley AJ (2011) Life at the leading edge. Cell 145:1012-1022.

Seidl AH, Rubel EW, Harris DM (2010) Mechanisms for adjusting interaural time differences to achieve binaural coincidence detection. J Neurosci 30:70-80.

Seidl AH, Rubel EW, Barría A (2014) Differential conduction velocity regulation in ipsilateral and contralateral collaterals innervating brainstem coincidence detector neurons. J Neurosci 34:4914-4919.

Sells MA, Pfaff A, Chernoff J (2000) Temporal and spatial distribution of activated Pak1 in fibroblasts. J Cell Biol 151:1449-1458.

Siegel GJ, Agranoff BW (1999) Basic neurochemistry. Philadelphia: Lippincott Raven.

Slack-Davis JK, Eblen ST, Zecevic M, Boerner SA, Tarcsafalvi A, Diaz HB, Marshall MS, Weber MJ, Parsons JT, Catling AD (2003) PAK1 phosphorylation of MEK1 regulates fibronectin-stimulated MAPK activation. J Cell Biol 162:281-291

Snaidero N, Möbius W, Czopka T, Hekking LH, Mathisen C, Verkleij D, Goebbels S, Edgar J, Merkler D, Lyons DA, Nave KA, Simons M (2014) Myelin membrane wrapping of CNS axons by $\mathrm{PI}(3,4,5) \mathrm{P} 3$-dependent polarized growth at the inner tongue. Cell 156:277-290.

Snaidero N, Velte C, Myllykoski M, Raasakka A, Ignatev A, Werner HB, Erwig MS, Möbius W, Kursula P, Nave KA, Simons M (2017) Antagonistic functions of MBP and CNP establish cytosolic channels in CNS myelin. Cell Rep 18:314-323.

Tang Y, Zhou H, Chen A, Pittman RN, Field J (2000) The Akt proto-oncogene links Ras to Pak and cell survival signals. J Biol Chem 275:9106-9109.

Tomassy GS, Berger DR, Chen HH, Kasthuri N, Hayworth KJ, Vercelli A, Seung HS, Lichtman JW, Arlotta P (2014) Distinct profiles of myelin distribution along single axons of pyramidal neurons in the neocortex. Science 344:319-324.

Velumian AA, Samoilova M, Fehlings MG (2011) Visualization of cytoplasmic diffusion within living myelin sheaths of CNS white matter axons using microinjection of the fluorescent dye Lucifer Yellow. Neuroimage 56:27-34.

Wahl SE, McLane LE, Bercury KK, Macklin WB, Wood TL (2014) Mammalian target of rapamycin promotes oligodendrocyte differentiation, initiation and extent of CNS myelination. J Neurosci 34:4453-4465.

Yamamura T, Konola JT, Wekerle H, Lees MB (1991) Monoclonal antibodies against myelin proteolipid protein: identification and characterization of two major determinants. J Neurochem 57:1671-1680.

Ye DZ, Field J (2012) PAK signaling in cancer. Cell Logist 2:105-116.

Zenke FT, King CC, Bohl BP, Bokoch GM (1999) Identification of a central phosphorylation site in p21-activated kinase regulating autoinhibition and kinase activity. J Biol Chem 274:32565-32573.

Zhang S, Han J, Sells MA, Chernoff J, Knaus UG, Ulevitch RJ, Bokoch GM (1995) Rho family GTPases regulate p38 mitogen-activated protein kinase through the downstream mediator Pak1. J Biol Chem 270:23934-23936.

Zhang Y, Chen K, Sloan SA, Bennett ML, Scholze AR, O'Keeffe S, Phatnani HP, Guarnieri P, Caneda C, Ruderisch N, Deng S, Liddelow SA, Zhang C, Daneman R, Maniatis T, Barres BA, Wu JQ (2014) An RNA-sequencing transcriptome and splicing database of glia, neurons, and vascular cells of the cerebral cortex. J Neurosci 34:11929-11947.

Zuchero JB, Fu MM, Sloan SA, Ibrahim A, Olson A, Zaremba A, Dugas JC, Wienbar S, Caprariello AV, Kantor C, Leonoudakis D, LariosaWillingham K, Kronenberg G, Gertz K, Soderling SH, Miller RH, Barres BA (2015) CNS myelin wrapping is driven by actin disassembly. Dev Cell 34:152-167. 